COMPTES RENDUS

\title{
SCIENCE ET PHILOSOPHIE
}

The Cambridge Companion to Plotinus. Ed. by Lloyd P. Gerson. Cambridge, Cambridge University Press, 1996. 15 × 22,8, xIII-462 p., bibliogr., index (Cambridge Companions to philosophy).

Dans ce gros volume destiné, comme tous ceux de la collection, avant tout aux étudiants en philosophie et aux amateurs éclairés qui veulent faire le point sur l'état actuel de la recherche sur un auteur, tous les aspects essentiels de la pensée de Plotin et des débats interprétatifs sur son œuvre sont effectivement présentés. Lloyd $P$. Gerson, responsable du volume et qui signe l'introduction, a marqué le volume de son empreinte en faisant en sorte que chaque auteur, en présentant un aspect de la pensée de Plotin, s'attache aussi à la filiation et à l'originalité de Plotin par rapport à Platon et à la genèse du néo-platonisme. Gerson lui-même, dans son introduction, interroge ce concept de néo-platonisme, sa signification et sa pertinence puisqu'aussi bien Plotin ne s'est pas considéré comme néo-platonicien mais bien comme le continuateur et l'exégète de Platon. Or, se dire platonicien, c'est accepter comme éternellement vrais certains principes de la philosophie platonicienne, par exemple l'antériorité fondatrice de l'un sur le multiple, l'existence de vérités éternelles portant elles-mêmes sur des entités éternelles, le caractère paradigmatique de ces réalités éternelles et intelligibles par rapport aux êtres sensibles ou bien encore le caractère spirituel du soi authentique et le rôle de l'amour dans la remontée vers Dieu. Mais c'est aussi considérer que Platon n'a pas dit le tout de tout, qu'on doit confronter les dialogues platoniciens avec leur exégèse ultérieure, y compris ce que dit Aristote de l'enseignement oral; c'est, enfin, prolonger Platon sur ce qu'il n'a fait qu'ébaucher, comme le fait Plotin en III, 7 sur l'éternité et le temps. C'est d'ailleurs une des richesses de ce volume que d'articuler soigneusement l'originalité des thèses plotiniennes sur la tradition qui leur donne sens. En revanche, une plus grande réserve s'impose sur les tentatives (par exemple, celle de Sara Rappe p. 250274) qui, à la suite de Rorty, veulent voir dans Plotin un précurseur de Descartes et dans ce Plotinus cartesianus, un théoricien de la connaissance de soi et de la naissance moderne du sujet.

Une première série d'études analyse les divers aspects de la métaphysique plotinienne : son enracinement dans le platonisme (Maria Luisa Gatti), la métaphysique de l'Un (John Bussanich), l'ordre hiérarchique du réel (Dominic O'Meara), le rap- 
port entre l'âme et l'intellect (Henry Blumenthal), l'essence et l'existence (Kevin Corrigan). Une série suivante porte sur les questions de physique : la nature de la réalité physique (Michael Wagner), la matière et le mal et subséquemment la théodicée plotinienne (Denis O'Brien), l'éternité et le temps (Andrew Smith), la connaissance et son objet (Eyjolfur Kjalar Emilsson). Enfin, un troisième groupe d'études porte sur des questions qu'on pourrait appeler morales au sens large : la connaissance de soi et la subjectivité (Sara Rappe), le rapport de l'âme et du corps (Stephen Clark), la liberté humaine (Georges Leroux), l'éthique plotinienne caractéristique d'une éthique pour un sage de l'antiquité tardive (John Dillon), la question du langage (Frederic Schroeder), la causalité du premier principe et la difficulté de dériver le multiple de l'Un sans multiplier les médiations (Cristina d'Ancona Costa) et, enfin, une admirable étude de John Rist qui étudie la question controversée du néoplatonisme chrétien (ou de l'influence spécifique de Plotin sur les auteurs chrétiens des $I v^{e}$ et $v^{e}$ siècles) en adoptant sur ce sujet une position minimaliste.

Le tout est suivi d'une bibliographie de vingt-deux pages mais où manquent trop de travaux importants des dernières années, écrits en français il est vrai - ainsi, les articles de Jean-Louis Chrétien (sur l'analogie chez Plotin ou sur « Le bien donne ce qu'il n'a pas "), le livre de Jérôme Laurent sur Les Fondements de la nature selon Plotin, les articles de Jean-Marc Narbonne sur la question de la causa sui...; de même que certains travaux évoqués dans le cours des articles (l'étude d'Alain Eon sur les principes de l'exégèse plotinienne ou Hadot 1994, probablement la traduction commentée publiée au Cerf du traité 9) sont absents de cette bibliographie.

Viennent ensuite un index des passages cités (de Plotin ou d'autres auteurs antiques) et, enfin, un index des noms et des principaux thèmes traités, index qu'on souhaiterait plus complet notamment pour les thèmes (par exemple, pas de référence à immanence, un thème qui intervient pourtant à de nombreuses reprises dans la présentation de la métaphysique plotinienne).

Cet ouvrage clair, remarquablement informé, précis sans être pédant, est à recommander largement au-delà du cercle des étudiants de philosophie anglophones.

Jacqueline LAGRÉE

Maurice Clavelin, La Philosophie naturelle de Galilée. Essai sur les origines et la formation de la mécanique classique. Paris, Albin Michel, 1996. 12,5 × 19, 511 p., index, bibliogr. (Bibliothèque de l'Évolution de l'humanité, 23).

Un ouvrage majeur connaît enfin une nouvelle édition, dans un format dont la pagination respecte celle de 1968 , avec une bibliographie complète des études galiléennes contemporaines. Cette publication met un terme à l'intolérable situation de pénurie qui touchait un authentique classique de l'histoire des sciences, voire de l'histoire de la philosophie.

S'agissant d'un ouvrage aussi connu, il sera moins question ici d'en analyser les contenus avec précision que d'indiquer les raisons de son actualité et de sa pertinence. Publier cet ouvrage en 1996 est déjà en soi un acte dont il faut évaluer la 
signification et la valeur de résistance ou d'opposition face à la prise en charge de l'histoire des sciences par ce que l'on nommera, en général, une approche sociologique. Maurice Clavelin lui-même se charge de préciser, dans une préface à la seconde édition, le sens de ce geste, en revenant aux sources de sa propre compréhension de l'histoire des sciences, "dont la tâche est de rendre intelligible l'invention et le développement de théories destinées à maîtriser des données objectives toujours plus complexes, [et qui] ne saurait atteindre son but sinon en retrouvant les voies de cette invention et de ce développement " (p. II). La référence est ici à une reconstruction interne de l'acte d'inventer, ce dernier étant posé comme l'objet propre de l'histoire des sciences, par opposition aux « marges " où se mouvraient tout à la fois l'histoire « érudite " et l'analyse contextualiste : "Soutenir que dans le contexte socioculturel se trouve la clef des problèmes et des concepts dont dépend le développement de la science, c'est donc à nouveau demeurer sur ses marges [nous soulignons]; c'est en même temps revenir à un usage passablement obscur de l'explication causale, et pour finir diluer la connaissance scientifique parmi les autres formes de l'activité humaine » (ibid.). Clavelin cite alors en note l'ouvrage qu'il a codirigé avec Raymond Boudon, Le Relativisme est-il résistible? (Paris, Presses universitaires de France, 1994), ouvrage dans lequel il avait eu l'occasion d'affirmer ses positions face à la sociologie montante (voir « L'histoire des sciences face à la sociologie des sciences ", in ibid., p. 227-247). La sociologie dite du " programme fort » est, dans ce dernier ouvrage, distinguée avec soin d'une " sociologie scientifique de la connaissance, celle qui sans se prendre pour la science de la science s'efforce tout simplement de procéder à la façon d'une science » (ibid., p. 247). On peut regretter que cette distinction ne soit pas aussi bien marquée dans la seconde édition de La Philosophie naturelle de Galilée. Clavelin ne justifie pas, en particulier, la notion de " marge " et son application à l'approche sociologique en général. Or, il faudrait distinguer, comme cela est le cas dans l'ouvrage de 1994, entre deux façons de comprendre l'activité de la sociologie des sciences. Ainsi la " sociologie scientifique » (de type durkheimien), qui n'est pas condamnée par Clavelin, travaille à l'élucidation de ce qui a pu motiver telle ou telle thèse portant sur un moment historique (par exemple le mythe de la « révolution scientifique »). Son point de vue est externe, certes, mais ne prétend pas poser les déterminations qu'elle élabore en conditions de possibilité des théories scientifiques. En ce sens, sa pratique est légitime et se distingue du "programme fort » de la sociologie des sciences. L'histoire des sciences, quant à elle, se tourne moins vers les motifs et raisons des interprétants de l'histoire que vers l'histoire elle-même, conçue comme un ensemble de données «objectives ». Le relativisme proprement dit, celui du programme fort, en prétendant donner le sens des faits scientifiques eux-mêmes à partir du contexte où ils se produisent, ne dispose, dans le propos de Clavelin, d'aucune légitimité : il est, littéralement, «dans » les marges, c'est-à-dire qu'il se meut à la périphérie de la science. Les tenants de ce programme (l'auteur vise essentiellement David Bloor en 1994) affirment précisément que la science tient son principe de ce qui l'entoure, du tissu sociohistorique qui la détermine en lui imposant un type de discours spécifique. La science est agie par ce qui est nommé ici ses « marges ». On saisit mieux alors l'intention de cette publication. Moins destinée à combler la pénurie qu'à occuper le terrain de l'histoire des sciences, elle est la promotion, en acte, d'une méthode. 
Contre ce point de vue de la détermination sociale qui méconnaît en effet trop souvent la spécificité de l'objet scientifique, Clavelin fait valoir la singularité des idées en science. Ces dernières, tout en emportant une part d'idéologie, doivent être essentiellement comprises à l'intérieur du système qu'elles contribuent à façonner. Cette perspective systématique conduit Clavelin à minorer l'apport de ce qu'il considère comme la principale innovation des études galiléennes: l'analyse des manuscrits relatifs à la science du mouvement. Prenant acte des travaux de S. Drake, W. L. Wisan, P. Galluzi ou W. A. Wallace qui gravitent autour de la nécessité d'ordonner les manuscrits afin de restituer la chronologie exacte de la naissance des concepts galiléens, il rappelle la nature « hypothétique » (p. v) de telles recherches, leur préférant l'ordre d'exposition "raisonné », que représente le texte achevé des Discorsi. C'est en effet dans le texte tel qu'il est imprimé ou dans les essais composés et cohérents que sont les Juvenilia, que Clavelin voit se mettre en œuvre le cour de la pensée et de la philosophie galiléennes. Néanmoins, il pose une fois de plus, en page Ix, la question de l'appartenance de ces travaux à l'histoire des sciences. La thèse de Wallace, en particulier, selon laquelle Galilée n'aurait eu qu'à combiner certains éléments d'une logique héritée de l'aristotélisme zabarellien (la méthode dite du regressus démonstratif) avec le « modèle archimédien », ne semble pas permettre, pour lui, de trancher définitivement le conflit des interprétations relatives à la méthode galiléenne, tour à tour supposée en rupture ou en continuité avec l'héritage scolastique. Arguant de l'hétérogénéité entre le continuisme d'un Duhem et celui d'un Wallace, Clavelin insiste sur le fait que la continuité établie par le premier repose sur l'analyse des concepts et des problématiques internes à la « science moderne », tandis que celle qui est posée par le second ne fait que " trouver hors de l'œuvre la clef de la méthode », tout en prétendant faire naître la physique mathématisée dans une doctrine où le rôle de ces mathématiques est nul. Son argument ultime repose donc sur l'incohérence conceptuelle, l'irrationalité même de tels projets, dont l'envergure historique se déploierait comme en vain dans une contradiction initiale insoutenable.

L'analyse interne s'effectue en quatre parties qui sont autant de mises à l'épreuve de la méthode de l'histoire des sciences telle que Clavelin la conçoit, soit «Traditions et travaux de jeunesse » (p. 17-178), « Le copernicianisme et la science du mouvement » (p. 179-276), « Naissance de la mécanique classique » (p. 277-385) et «La raison et le réel » (p. 387-459). Dans la première partie, la logique interne de la progression articule la nécessité d'analyser les systèmes conceptuels qui se présentent à Galilée pour parvenir in fine à expliciter et à déplier les éléments de la méthode galiléenne. On est conduit insensiblement d'une logique du savoir à une autre, sans que soit mise en œuvre la problématique controversée de la « révolution scientifique » ou « intellectuelle ", mais en relevant à chaque fois les filiations et mutations qui affectent les méthodes dont Galilée hérite d'une antique tradition, dont il ne modifie pas la logique (la recherche de principes qui permettraient la déduction géométrique des lois établies est conforme, comme Clavelin le note à juste titre dans Le Relativisme est-il résistible, op. cit., art. cit., p. 234, au modèle déductif des Seconds analytiques), mais le contenu et les principes. Le chapitre I de la première partie est, en ce sens, remarquable, car en montrant nettement que la science aristotélicienne du mouvement se fond dans l'ordre cosmologique et en devient indissociabie (p. 31, Clavelin parle d'une " image cosmologique a priori »), 
l'analyse conceptuelle explicite largement la façon dont Galilée reçoit et perçoit la question du mouvement à l'intérieur d'un noyau cosmologique préconstitué où, par exemple (cf. p. 215), le repos et le mouvement circulaire, sortes d' « états naturels » (p. 219) prennent le pas sur le mouvement rectiligne uniforme. Le troisième moment de cette première partie, qui s'attache aux travaux de jeunesse, accentue encore l'absence de problématique de la rupture. Le Meccaniche, par exemple, unifient les traditions issues d'Archimède, du pseudo-Aristote et de la statique médiévale et parvient, en exploitant subtilement la notion de moment de descente, à un traitement général et neuf des notions de résistance et de puissance engagées dans un équilibre. L'indication d'un anéantissement du moment de descente sur une surface plane indique en creux l'existence d'un état où le mobile est indifférent au mouvement comme au repos, les conditions de l'équilibre entre puissance étant elles-mêmes anéanties. Clavelin, p. 164, prend acte, en pleine appropriation de la statique médiévale, d'une première dissociation entre mouvement et inclination, prédétermination au repos. Dans une certaine mesure, cette dissociation anticipe la découverte d'un mouvement rectiligne potentiellement infini sur une surface plane assimilée, comme cela sera le cas dans une fameuse expérience de pensée des Discorsi (Troisième journée, « Du mouvement naturellement accéléré », scolie du Problème IX, Proposition XXIII) à une absence des " causes extérieures d'accélération et de ralentissement » qui permet au degré de vitesse acquis de façon indélébile d'exprimer sans contrainte sa faculté de transporter le mobile à l'infini suivant une vitesse uniforme.

Il faut noter pour finir que la science galiléenne s'amende par une suite d' « initiatives intellectuelles » qui disent assez le primat de la réflexion autonome dans l'élaboration de la mécanique classique. En accentuant, c'est-à-dire en isolant le corps des réponses, ajustements, emprunts et rectifications que Galilée fait à l'ensemble des anciennes doctrines, Clavelin répondait, en 1968, à la thèse de Paul Tannery et d'Alexandre Koyré concernant la structure platonicienne du savoir galiléen. À ceux-là, qui se maintenaient dans l'horizon de l'histoire des sciences, le présent ouvrage offrait un interlocuteur de choix dans l'analyse de la méthode de Galilée. Le champ s'est aujourd'hui déplacé et ce manifeste d'interprétation systématique ne trouvera sans doute, chez les tenants du programme sociologique fort, qu'incrédulité et mépris. On ne voit cependant pas comment la plupart des métamorphoses conceptuelles qui se dévoilent ici pourraient être assignées à un quelconque jeu des puissances sociales. S'agissant des transformations subies par les concepts mécaniques, l'analyse de Clavelin, même si elle choisit de privilégier certaines filiations intellectuelles en les isolant, parvient du moins à circonscrire, malgré les charges provocantes de la préface, l'espace intellectuel qui devrait être celui de l'histoire des sciences.

Fabien Chareix

Jocelyn Benoist, Kant et les limites de la synthèse. Le sujet sensible. Paris, Presses universitaires de France, 1996. $15 \times 21,5,345$ p., bibliogr., index (Épiméthée).

À l'origine de ce livre se trouve la volonté d'interroger la notion de «subjectivité ", telle qu'elle se donne à penser dans la Critique de la raison pure. Or, 
cette « subjectivité " semble pour le moins problématique dans un texte qui semble se débarrasser de la figure postcartésienne du sujet. En effet, le sujet y est réduit soit à un objet empirique (l'objet du sens interne), qui parce qu'il est objet n'est plus sujet, soit au sujet transcendantal $=X$, qui est certes un sujet, mais un sujet vide ou une abstraction de sujet, et qui, ce faisant, représente la destruction même du concept traditionnel de sujet ( Le sujet transcendantal se présente comme une machine de guerre contre les métaphysiques de la subjectivité », p. 73). Le sujet semble donc inassignable dans la Critique de la raison pure.

Cette alternative, maintes fois soulignée par les commentateurs, est la conséquence directe de la problématique de la Critique de la raison pure, qui est celle de la fondation de l'objectivité, dans laquelle le sujet ne semble pas avoir sa place, si ce n'est en tant que fondement de l'objectivité, c'est-à-dire comme sujet transcendantal. Il ne peut pas y avoir de « philosophie du sujet » dans une perspective transcendantale, qui a pour interrogation les structures de la transcendance; il n'y a de philosophie que de l'objet, et ce n'est que dans cet horizon de l'objet que peut apparaître, en creux, le sujet : "La question du sujet est indissociable du problème de l'objet " (p. 73). De ce fait, le sujet n'est rien de donné. Mais, et telle est l'intuition de départ de Jocelyn Benoist, « il paraît douteux que le sujet puisse jamais être réduit entièrement à une forme pure " (p. 13). Cependant, pour parvenir à saisir comment la première Critique se trouve être le lieu d'une redétermination de la notion de sujet, il fallait reposer une nouvelle fois, et depuis le début, la question de l'objectivité, celle de ses formes et de ses conditions a priori.

Cette relecture de la problématique transcendantale occupe toute la première partie de l'ouvrage intitulée «La synthèse ». Elle nous présente une remontée de « la question de l'objet », ou de l'objectivité en général, vers le « sujet » qui en est le fondement. Cette remontée conduit en dernier ressort l'Auteur à une réflexion sur la notion de synthèse (chap. v), en tant que c'est en elle que se constitue l'objet, comme unité de sens. La synthèse constitue en effet l'objet, en ce que c'est en elle que ce dernier s'unifie. Mais la synthèse doit en plus être supportée par l'aperception transcendantale, ou «conscience de soi », qui fait que la représentation de l'objet est pour moi : «La représentation n'est pensée qu'en tant qu'elle s'aperçoit elle-même » (p. 150). Cette aperception désigne le fait d'être conscient, ou encore, selon la formule de Kant, le fait d'avoir « la représentation qu'une représentation est en moi »; autrement, il y aurait en moi des représentations dont je ne serais pas conscient : «Ce qu'énonce le “ Je pense ”, c'est l'aperceptibilité de la représentation [...], au sens où la représentation, lieu de l'apparaître, ne peut elle-même apparaître et manifester son sens de représentation qu'à condition d'être perceptible " (p. 151). C'est là qu'apparaît le sujet. Ce qui manifeste la présence d'un sujet, c'est donc que toutes mes représentations doivent pouvoir m'apparaître comme mes représentations. L'unité de l'objet appelle, en effet, l'unité de la conscience, qui se présente comme une identité. Au fondement de la synthèse se trouve donc «l'identité personnelle " qui, seule, peut rendre raison de l'unité qu'elle confère à l'objet.

C'est là que les difficultés apparaissent. En effet, «si l'identité est identité de la synthèse, cela ne signifie pas qu'il n'y ait pas [...] synthèse d'une identité de la synthèse » (p. 154). Et, « pour que la synthèse (la conceptualisation) soit possible il faut qu'il y ait synthèse originaire qui déploie la possibilité même de la synthèse, en tant que possibilité en général d'une liaison, ce que Kant appelle la " synthèse transcen- 
dantale" " (p. 157). Cela appelle une interrogation sur les conditions de la synthèse. Or, et c'est sur ce point que se révèlent la nouveauté et l'originalité profondes de l'interprétation de Benoist, cette « synthèse de la synthèse " comporte des limites, n'est pas clôturable. Dans ces limites se joue l'émergence d'un sujet, en un sens nouveau, appelé ici pour cette raison une "subjectivité " plutôt qu'un sujet. Voilà qui appelait une deuxième partie, intitulée "La thèse " qui, "au-delà de la synthèse » pose une nouvelle fois, et en des termes radicalement nouveaux, « la question du sujet " (p. 162).

Les « limites de la synthèse » nous mettent en présence d'un " sujet sensible ", parce que ce qui résiste à la synthèse, ce qui demeure irréductible à elle, est de l'ordre de l'affection, de la réceptivité, ou encore de la sensibilité : « Dans le sensible, il n'y a pas que de la transcendance ", il y a toujours aussi un "fond d'immanence qui est celui de la réceptivité du divers, en tant que pur fait d'être-affecté " (p. 163). Cela impliquait derechef une relecture de l'Esthétique transcendantale, qui est le niveau propre de la sensibilité. Ce que met en évidence Benoist, c'est qu'il y a une passivité originaire et irréductible à la synthèse, passivité qui résiste à la reprise absolue de soi, c'est-à-dire à l'autoconstitution. "Cette béance originaire est celle de la synthèse par rapport à elle-même, habitée qu'elle est par son autre, pure et simple thèse de la sensibilité $»$ (ibid.). C'est dans ce fond d'affectivité, d'irréductible passivité, que git, selon Benoist, la subjectivité.

Tout au long de la deuxième partie de l'ouvrage, il s'attache ainsi à montrer l'émergence et la teneur de cette subjectivité qu'il croit pouvoir déceler en deçà de l'objectivation. Cela impliquait de sa part une réévaluation du rôle de l'espace par rapport à celui du temps, sur lequel les commentateurs, depuis Heidegger, avaient coutume d'insister. Une lecture serrée de l'interprétation heideggerienne fait d'ailleurs l'objet du premier chapitre, qui aboutit à la conclusion d'une irréductibilité de l'espace au temps (mais aussi, symétriquement, du temps à l'espace), l'un et l'autre ne pouvant être reconduits «à l'unité originaire d'une "réceptivité " qui serait d'emblée et sans reste temporelle $»$ (p. 226). Cette revalorisation de l'espace (chap. II) tient donc à « l'insuffisance du temps à constituer à lui seul le niveau esthétique » (p. 206). En effet, l'espace apparaît comme « l'horizon indépassable de "l'intuitivité" comme telle » (p. 207). La spatialisation constitue le premier niveau de la donation, indépassable et ineffaçable : «Le sensible comme tel - le sensible "pur" - ne peut m'être donné que sous le régime de l'extensivité, dans la fondamentale dimensionalité de son être-donné » (p. 224).

On touche, avec la sensation, à la dimension phénoménologique de la subjectivité. Cette dernière apparaît et se constitue dans la sensation comme " être-affecté », et non pas comme ce qui préexisterait à la sensation et la rendrait possible. Ainsi, Benoist peut-il écrire : « La thèse du sujet [est] comme ancrée dans la passivité ellemême, en reste de l'activité de synthèse " (p. 248). Cela signifie qu'il y a quelque chose, dans « l'Esthétique transcendantale [qui] ne peut se résorber dans la Logique transcendantale », et qui est «l'affection » (p. 14). En d'autres termes, le décalage entre l'entendement et la sensibilité, qui sont les deux sources de la connaissance, ne peut jamais être complètement comblé, et c'est la force du texte kantien que de chercher à les faire tenir ensemble sans jamais en escamoter un au profit de l'autre. $\mathrm{La}$ « thèse » du sujet rend alors possible une relecture originale de la notion de « sens interne » (chap. Iv) : “ Nous sommes affectés " par" des objets et cette affec- 
tion nous fait apparaître à nous-mêmes comme étant affectés " (p. 276). Le moi est donc «ce que j'éprouve dans l'affection, en tant que je ne considère plus l'objet, mais l'affection elle-même » (p. 275). Voilà ce que signifie l'autoaffection : non pas affection de soi par soi, mais affection de l'affection, au sens où dans l'affection par l'objet, je fais l'expérience passive de ma propre affection, qui me révèle moi-même à moi-même.

C'est donc toujours dans l'horizon de la transcendance que se découvre le sujet, et qui plus est, en marge du processus d'objectivation : «Il n'y a pas un sujet qui précéderait la sensibilité qui serait sensibilité " de " ce sujet, il y a la subjectivité de l'homme fondée dans la sensibilité première de son rapport à l'objet, en tant que celle-ci, dans le rapport même à l'objet, ouvre le lieu d'un " contenu " du phénomène qui est irréductible à cet “objet" " (p. 282). Le donné transcendant n'est jamais totalement récupéré par le travail de synthèse qui le détermine catégorialement comme tel ou tel objet; il reste toujours un fond de passivité qui n'est pas effaçable, et ce fond est la subjectivité elle-même. En deçà du sujet transcendantal, qui vide le sujet de tout être, Benoist retrouve la subjectivité à un niveau phénoménologique, qui se trouve être la condition du processus d'objectivation.

Un des intérêts principaux de cet ouvrage, qui rend sa thèse particulièrement féconde, est d'être parvenu à effectuer une lecture "phénoménologique " de la Critique de la raison pure, en ce qu'elle utilise à la fois les horizons problématiques ouverts par la phénoménologie, ainsi que ses acquis conceptuels, et les interprétations phénoménologiques particulières qui ont été données de la philosophie kantienne, comme celle de Heidegger. Mais en même temps, à travers ce prisme phénoménologique, l'objectif est ici de revenir au texte kantien et, d'une certaine façon, de lui rendre justice, en montrant qu'il ouvre notre modernité philosophique quant à la question du sujet. Cette modernité est, entre autres, liée à ce qui est appelé, dans la conclusion, « l'anti-idéalisme » de Kant. L'anti-idéalisme qui caractérise l'interrogation kantienne sur la subjectivité tient à ce que le sujet ne peut plus être caractérisé par ses contenus subjectifs. Avec Kant, le « sujet " cesse d'être donné, cesse d'être une donnée, thématisable en et pour elle-même, pour s'apparaître et se révéler dans et par son activité constituante, que celle-ci soit théorique ou pratique : « Tout se passe comme si la disparition ou la relativisation du sujet comme " contenu " libérait " l'appel " constitutif de la subjectivité, d'abord déterminée non pas comme celle qui est telle ou telle chose, mais comme celle qui a à faire telle ou telle chose ${ }^{1}$. " Voilà qui fait apparaître un sens original de la subjectivité, subjectivité ouverte à et par la transcendance, et essentiellement caractérisée par ses « tâches ». On voit que, loin d'empêcher toute réflexion autour de la notion de sujet, le questionnement kantien ouvre au contraire un espace inédit d'interrogation. En cela se révèle précisément la modernité de la pensée kantienne, dont il n'est pas sûr d'ailleurs que la phénoménologie ait su prendre toute la mesure, et ce n'est pas le moindre mérite du livre de Jocelyn Benoist que de l'avoir soulignée avec beaucoup de clarté et d'intelligence.

Antoine RouLLÉ

1. Jocelyn BenoIst, «La subjectivité », in Notions de philosophie, dir. Denis KambouchNER, Paris, Gallimard (Folio. Essais), 1995. 
Jeremy BeNTHAM, De l'ontologie et autres textes sur les fictions. Texte anglais établi par Philip Schofield, trad. et comment. par Jean-Pierre Cléro et Christian Laval. Paris, Seuil, 1997. 11 × 18, 290 p., bibliogr., index (Points/Essais, 353).

Les auteurs de la présente édition bilingue de l'essai De l'ontologie de Jeremy Bentham (composé en 1814, et accompagné ici de quatre courts textes sur le même sujet, datant de 1813, 1814 et 1821) annoncent explicitement de grandes ambitions. Il s'agit d'abord de faire découvrir, derrière la catégorie éculée de l'utilitarisme, une pensée de l'ontologie, de la logique et du langage, qu'on peut résumer sous l'expression de théorie des fictions; ensuite, de montrer que cette théorie des fictions n'est pas autre chose que la clé de voûte de toute l'œuvre de Bentham; enfin, de suggérer que cette théorie peut s'avérer utile pour redéfinir le concept d'idéologie, dans le cadre d'une analyse des sciences de notre temps, et particulièrement des sciences humaines (p. 64-65). Quels sont donc les moyens mis au service de ces ambitions?

Le premier point fort de l'ouvrage est d'être le fruit d'une collaboration francobritannique. Philip Schofield, coéditeur général des Collected Works of Jeremy Bentham dans le cadre du Bentham Project de l'University College London (une vingtaine de volumes ont déjà paru), en a établi le texte anglais à partir des manuscrits de l'auteur. L'essai De l'ontologie avait certes déjà été publié en 1841 dans l'édition des ceuvres de Bentham établie par John Bowring, mais le texte avait alors été imprécisément retranscrit, et même tronqué sur certaines questions touchant à la religion. Le nouveau texte corrige ces défauts en suivant les principes de la nouvelle édition scientifique des Collected Works, au sein de laquelle il sera réédité plus tard. En attendant, le public francophone a l'avantage de la primeur du travail de Schofield. Du côté français, les traducteurs n'en sont pas à leur première contribution à l'introduction de la pensée de Bentham en France. Jean-Pierre Cléro avait déjà donné une traduction du Fragment sur le gouvernement, suivi du Manuel de sophismes politiques (Paris, LGDJ, 1996). Quant à Christian Laval, il avait analysé la théorie des fictions dans son livre Jeremy Bentham. Le pouvoir des fictions (Paris, Presses universitaires de France, 1994). Dans la présente édition, le travail commun de Cléro et de Laval donne lieu à un ouvrage très dense, composé de quatre parties : une longue introduction, les textes de Bentham et leur traduction, un glossaire très fouillé et un dossier de textes d'auteurs postérieurs à Bentham.

L'introduction des traducteurs expose tout d'abord, sur une soixantaine de pages, la genèse et la structure de la théorie des fictions. Sans reprendre leur démonstration, qui repose sur de très intéressantes distinctions entre «fiction», «fallace», " entité fictive ", etc., trois points de cette introduction méritent quand même d'être soulignés, pour leur originalité. En premier lieu, on trouvera en quelques pages étonnamment synthétiques une histoire de la problématique des fictions depuis John Locke, insistant tout particulièrement sur le rôle de David Hume. Ces aperçus sont d'ailleurs aussi suggestifs pour la lecture de Hume que pour celle de Bentham, et l'on se souviendra que Cléro a récemment publié un Hume (Paris, Vrin, 1998). En deuxième lieu, et sans doute s'agit-il là de l'apport le plus décisif de cette introduction, les traducteurs proposent d'interpréter la théorie des fictions à la lumière de deux paradigmes mathématiques, la méthode des fluxions (reprise d'Isaac Newton) et la théorie des chances (où il est question, à nouveau, de l'influence de Hume). Il 
en résulte de nouvelles perspectives sur l'idée benthamienne, bien connue, d'un calcul des plaisirs et des peines. En dernier lieu, il faut féliciter les traducteurs d'avoir le souci de souligner constamment les difficultés du projet de Bentham, tant d'un point de vue théorique que pratique. Le parti qu'ils ont pris d'évoquer, là encore, la figure de Hume, pour faire ressortir la spécificité des thèses benthamiennes, s'avère convaincant : Bentham a-t-il, comme il le voulait, dépassé le scepticisme humien? Son radicalisme politique n'est-il pas utopique face au conservatisme humien?

Si l'introduction des traducteurs expose la théorie des fictions de façon synthétique, les textes de Bentham offrent, quant à eux, l'image d'une pensée en train de se chercher, de se préciser elle-même. Des classifications sont posées, puis reprises; des bifurcations sont esquissées. Plusieurs notes de Bentham montrent d'ailleurs qu'il aurait souhaité approfondir certains points de ses manuscrits ("Faire une recherche sur les sphéroïdes », p. 93, et même « Examiner si c'est exact », p. 105). La traduction sait rendre ces tours et détours, sacrifiant l'élégance du style à une exigence, tout à fait légitime en l'occurrence, de littéralité. En ce qui concerne le vocabulaire, il faut d'abord souligner de façon très générale, que le principe d'une collection bilingue possède ce très grand avantage de soulager les traducteurs de la lourde responsabilité d'être l'unique mode d'accès au texte original. La traduction devient alors en quelque sorte un dialogue avec l'original, les deux versions se clarifiant l'une l'autre. Reste que, même en bilingue, certaines options de traduction doivent être prises. Pour prendre l'exemple le plus central, comment traduire le terme-clé de fictitious? Traduire par « fictif» a le désavantage d'avoir la connotation de "trompeur ", alors que toute fiction, au sens de Bentham, n'est pas forcément une tromperie. Les traducteurs expliquent (p. 197) qu'ils ont malgré tout choisi cette option comme un moindre mal. Traduire par un terme ancien comme "ficticieux », ou par un terme éloigné comme «symbolique», aurait, on en conviendra aisément, été moins satisfaisant encore. D'autres choix de traduction, pour n'en être pas moins subtils, s'imposent, eux, indiscutablement. Par exemple, stock of language est rendu par «capital de la langue ", en conformité avec le vocabulaire économique de l'époque (p. 79). Ou encore, expectation est traduit par « espérance », parce qu'il s'agit du sens mathématique du mot (p. 61).

Vient alors un glossaire des termes utilisés par Bentham. Il occupe une soixantaine de pages, et il est organisé thématiquement en sections et sous-sections, ce qui en fait un véritable exposé méthodique de la théorie des fictions. Les analyses de l'introduction y sont approfondies - ce qui entraîne, il faut le dire, quelques redites -, tandis que des concepts nouveaux sont introduits. C'est dans le glossaire, par exemple, que la méthode dite de la " paraphrase », si nécessaire pour disqualifier les fictions trompeuses, est expliquée au lecteur. C'est également dans le glossaire que le rapport entre Bentham et Aristote, décisif pour définir le terme d'ontologie, est précisé. Le glossaire sert également à mieux faire ressortir, voire à justifier, certains choix de traduction. De plus, une quinzaine de très courts textes de Bentham, issus d'autres ouvrages, illustrent ce glossaire. Leur présence est bienvenue, dans la mesure où non seulement ils illustrent, mais encore où ils servent à donner au lecteur une idée de la récurrence des concepts dans l'œuvre de Bentham, et donc à en pressentir la systématicité.

Enfin, les traducteurs proposent un petit dossier d'une quinzaine de pages, composé de textes d'auteurs postérieurs à Bentham, commentant sa philosophie : 
John Stuart Mill, Karl Marx, Michel Foucault, Jacques Lacan, et Willard V. O. Quine. Le dossier retrace ainsi chronologiquement, à très grands traits, les différentes étapes de la réception de Bentham. Il en ressort un Bentham assez fragmentaire, par exemple caricaturé par Marx en " épicier anglais ", ou décrit par Foucault comme «le Fourier d'une société policière ». En évoquant ainsi, à la fin du livre, ces lectures décisives et stimulantes, mais peu soucieuses de retrouver la signification d'ensemble du projet de Bentham, nul doute que les traducteurs ont voulu faire ressortir par contraste leurs nouvelles pistes de lecture. Leur but est clairement de dégager l'œuvre de Bentham de son rôle de repoussoir, pour la présenter comme une source d'inspiration positive.

En résumé, donc, le lecteur trouvera trois livres en un : un commentaire précis des thèses de Bentham sur les fictions, des hypothèses d'interprétation s'étendant à l'cuvre entière et soulignant ses enjeux les plus vastes, et un ensemble de traductions de termes-clés susceptibles de servir de base à la traduction d'autres textes benthamiens. De quoi ouvrir des perspectives, quand on sait que les Collected Works annoncent plus de soixante volumes!

Michaël Bızıou

Georg Wilhelm Friedrich Hegel, Premiers écrits (Francfort 1797-1800). Textes réunis, introd., trad. et annot. par Olivier Depré, préc. de Sur l'antijudaïsme et le paganisme du jeune Hegel, par Robert Legros. Paris, Vrin, 1997. 13,5 × 21,5, 480 p., bibliogr., index (Bibliothèque des textes philosophiques).

Même si le titre choisi pour cette édition (Premiers écrits) des écrits de Francfort (1797-1800) de Hegel n'est pas précis (le jeune philosophe avait bien entendu déjà écrit auparavant, notamment à Tübingen et à Berne), l'édition elle-même est sans aucun doute la plus complète que nous ayons, en français, des textes de cette période. La bonne volonté et le sérieux d'Olivier Depré, le jeune chercheur au Fonds national belge auquel nous devons l'essentiel du travail accompli, ne pouvant être mis en cause, les lecteurs français et surtout, parmi ceux-ci, les amateurs de Hegel auraient eu là une véritable occasion de se réjouir - si leur joie ne se trouvait pas tempérée et même, parfois, gâchée, pour des raisons sur lesquelles nous reviendrons ci-dessous.

Mais il faut d'abord souligner les mérites du travail accompli par Depré. La tâche entreprise était, de fait, très difficile : l'édition critique de ces textes en allemand, préparée au Hegel-Archiv de Bochum (RFA), n'ayant pas encore été publiée, il devait s'appuyer sur des éditions plus ou moins limitées, comme celle, déjà ancienne, de Hermann Nohl (Hegels theologische Jugendschriften, Tübingen, Mohr, 1907), ou encore celles, plus récentes, de la maison Suhrkamp (Frühe Schriften, éd. par E. Moldenhauer et K. M. Michel, Francfort, 1971) ou de Werner Hamacher (Der Geist des Christentums, Francfort, Ullstein, 1978). Le terme d' « écrits » luimême contribue à nous cacher la vraie difficulté : il ne s'agit pas ici d'écrits destinés à la publication, mais d'un mélange de fragments, dont l'ordre reste à établir 
par le spécialiste en déchiffrement de manuscrits, ou par l'interprète. Si Nohl essaya de constituer, à partir des fragments qu'il avait à sa disposition, un texte suivi et cohérent - quitte, bien sûr, à ajouter un coup de main arbitraire à ce que le philosophe avait laissé inachevé -, les éditeurs modernes ont plutôt tendance à jouer la transparence, c'est-à-dire à mettre en évidence les lacunes et les ruptures. Même si les volumes de l'édition critique consacrés aux écrits de Francfort ( 2 et 5) ne sont pas encore disponibles, la conception et la stratégie qui en dirigent la composition sont déjà connues, et nous savons qu'elle ira sans doute plus loin encore dans la démarche décrite ci-dessus : chaque fragment sera présenté séparé des autres, selon le strict ordre chronologique. Depré, connaissant ces principes et les débats dont ils se sont dégagés, entend les suivre et les appliquer, avant même les éditeurs du Hegel-Archiv, aux textes de Francfort. Il le fait bien évidemment à ses risques et périls : lorsque l'édition critique sera achevée, il faudra déterminer si certains de ses choix et de ses décisions doivent être revus. Mais jusqu'alors, il serait injuste de l'accuser - pour avoir été plus rapide que l'équipe de chercheurs allemands.

Il est cependant regrettable que Depré - justement en raison de son zèle - ait parfois abandonné les grands principes de l'édition critique; et d'abord, pour ce qui concerne les notes. L'édition du Hegel-Archiv est toujours restée très classique, voire conservatrice, en cette matière, se contentant de donner les documents et de vérifier citations et emprunts, directs ou indirects, de Hegel. Elle s'est limitée à une stricte recherche de sources, philosophiques ou autres. La tentation est grande d'aller plus loin, et de citer tel ou tel article de la littérature secondaire, en raison de son apparente pertinence. Si les débutants apprécieront peut-être, reconnaissants pour telle ou telle suggestion de lecture, les spécialistes trouveront sans aucun doute certains des choix, peut-être même la plupart d'entre eux, peu concluants, voire même insatisfaisants. Depré, ici, est tombé dans un piège. Il peut d'autant plus facilement être critiqué que sa maîtrise de la littérature secondaire n'est pas toujours tout à fait à la hauteur de la tâche ambitieuse qu'il s'est proposée. Quelques exemples suffiront : sur Hegel et l'économie politique ou, plus précisément, sur la lecture par Hegel de sir James Steuart, Depré ne cite que Paul Chamley. Celui-ci fut, certes, un pionnier en la matière, fort estimé dans la discipline. Mais tant d'encre a coulé depuis en ce domaine, dans toutes les langues majeures utilisées par les spécialistes de Hegel, qu'il semble difficile de s'en tenir à Chamley. Sur les fragments politiques, notamment pour tout ce qui concerne leur rapport à la situation existant alors dans l'État du Würtemberg, on cherchera en vain les articles de Hans-Christian Lucas, pourtant l'un des spécialistes de la question. Les mêmes textes contiennent aussi bien des allusions à la politique britannique. Le rapport de Hegel aux pays anglo-saxons a récemment attiré beaucoup d'attention. Mais, étrangement, les notes de Depré restent muettes à ce sujet. Klaus Düsing, pourtant l'un des meilleurs connaisseurs de la jeunesse du philosophe, ne figure même pas dans la bibliographie; et le beau livre de Myriam Bienenstock sur la politique du jeune Hegel, qui est même en français, n'a pas eu plus de chance.

Plus grave encore que ces points de bibliographie, qui touchent pourtant aussi au contenu des annotations, semble être la décision d'intercaler dans le texte même de Hegel (par ex., p. 170-172) les comptes rendus que certains commentateurs du $\mathrm{XIX}^{\mathrm{e}}$ siècle ont fait de fragments perdus par la suite. Ces comptes rendus sont imprimés dans le volume avec une marge un peu plus large que celle utilisée pour le texte 
de Hegel, mais cela ne suffit guère pour distinguer entre texte et commentaire. Une distinction beaucoup plus nette aurait été nécessaire, surtout quand il s'agit d'un commentateur comme Rudolf Haym, dont nous savons aujourd'hui qu'il fut loin d'être innocent, et qu'il eut ses propres options.

Énoncées en pleine conscience de la difficulté de la tâche entreprise par Depré et non dans un esprit mesquin ou hostile, ces remarques critiques se veulent constructives et encourageantes, répondant, en toute confraternité de recherche, à une demande formulée par Depré lui-même, pour « une éventuelle amélioration future » (p. 91) de l'ouvrage. L'essai de Robert Legros, qui ouvre le volume, me semble beaucoup plus problématique. L'expression peu nuancée, dans le titre lui-même « l'antijudaïsme [...] du jeune Hegel » - est pour le moins maladroite : elle évoque, et semble confirmer, les accusations contre Hegel soulevées autrefois par André Glucksmann par exemple ( Les Maîtres Penseurs ») -, des accusations dont toute étude sérieuse sur Hegel devrait s'abstenir. Peut-on, aujourd'hui, parler sérieusement du rapport de Hegel au judaïsme sans tenir compte de la distinction, imposée par Shlomo Avineri il y a déjà trente-cinq ans (« A note on Hegel's view on Jewish emancipation », Jewish Social Studies, 25, 1963, p. 145-151), entre la présentation, par le jeune Hegel, du judaïsme «biblique », et l'engagement du Hegel de la maturité pour l'émancipation sociale et politique des juifs? Est-il juste et équitable de parler de l'un sans parler de l'autre? La stratégie de Legros, qui consiste à " disculper » le jeune Hegel en utilisant Lévinas - et en concluant avec Heidegger est, en tout cas, excentrée par rapport aux nombreux et sérieux articles consacrés à cette même question. Ce n'est pas le lieu, ici, de la critiquer adéquatement. Il suffira d'ajouter qu'un essai aussi personnel que celui de Legros aurait, peut-être, pu servir de postface, pour ouvrir une discussion sur le sujet. Comme préface à une édition qui se veut critique, il n'est certainement pas approprié.

Norbert WASZEK

The Correspondence of Michael Faraday. Vol. 2 : 1832-December 1840. Letters 525-1333. Ed. by Frank A. J. L. JAMES. Londres, The Institution of electrical engineers, $1993.15 \times 23,5$, LV-807 p., bibliogr., index.

In diesem zweiten Band des Briefwechsels von Michael Faraday wurden dieselben Editionsprinzipien befolgt wie in Band 1 (vgl. dazu die Besprechung in Revue de synthèse, IV $V^{c}$ S., 1-2, 1994, S. 231-233). In den Jahren nach 1830 erreichte Faraday die Höhe seines Ruhmes. Entsprechend umfangreicher wurde seine Korrespondenz, und da mit seiner eigenen Bedeutung und mit derjenigen seiner Korrespondenten auch die Wahrscheinlichkeit zunahm, daß Briefe aufbewahrt wurden, ist die Zahl der erhaltenen und hier komplett veröffentlichten Briefe erheblich größer als in den Jahren zuvor. Gegenüber Band 1 stieg die durchschnittliche Zahl der Briefe pro Jahr von 25 auf 90.

Der Gewinn für den wissenschaftshistorisch interessierten Leser ist freilich geringer, als diese Zahlen vermuten lassen. Zwar werden rund $70 \%$ der Briefe hier zum 
ersten Mal veröffentlicht; bei vielen handelt es sich jedoch nur um inhaltlich belanglose und kurze Gelegenheitsbriefe. Zahlreiche wissenschaftlich wichtige Briefe wurden dagegen schon an anderer Stelle veröffentlicht, so z. B. die 30 Briefe aus der Korrespondenz mit Schönbein in der von Kahlbaum und Darbishire besorgten Edition (Basel und London, 1899); viele andere Briefe findet man bei Williams, Fitzgerald und Stallybrass (Selected correspondence of Michael Faraday, Cambridge, 1971).

Da kein einziger Brief als Faksimile abgebildet wird, ist es schwierig, die Qualität der Transkriptionen zu beurteilen. Angesichts der Tatsache, daß der Herausgeber in den französischen Briefen selbst geringfügige Schreibfehler wie " porcellaine » und " y at-il » mit einem « [sic] » versieht, stellt sich jedoch die Frage, ob andere, nicht kommentierte und viel gravierendere Fehler nicht erst durch falsches Abschreiben der Originale entstanden sind (z. B. «réunion de Members de l'académie» (Brief 662), "Monsieur et tres confrere » (678), « gesammtet » (statt gesammelt), "des coups électrique " (723), "cetre Societé " (744)). Bei Brief 807 wird ohne Kommentar als Ort « Freyberg » angegeben. Da die Stadt, in der Faraday sich zu der fraglichen Zeit befand, auf halbem Weg zwischen Vevey und Bern liegt, kann es sich jedoch nur um Fribourg/Freiburg handeln. In Brief 1113 wird ein «meeting of the german association " erwähnt, ohne daß der Herausgeber verrät, welche Gesellschaft sich da getroffen hat. Statt dessen wurde ins Personen- und Sachregister unter Bezug auf diese Stelle der lapidare und sprachlich falsche Eintrag « Deutsche Geschellschaft " aufgenommen (gemeint ist die 16. Versammlung der Gesellschaft Deutscher Naturforscher und Ärzte von 1838). Ähnlich kryptisch sind einige Angaben über die Standorte der benutzten Manuskripte : woher soll der Leser wissen, daß mit "The Bakken Manuscript » die Handschriftensammlung der Bakken Library in Minneapolis gemeint ist?

Der Wert dieser Briefedition liegt vor allem darin, daß hier nicht nur das wissenschaftliche Werk, sondern auch das tägliche Leben des englischen Physikers sehr detailliert und in großer Dichte dokumentiert wird. Jeder, der in Zukunft über Faraday arbeitet, wird darauf zurückgreifen müssen.

Andreas KLEINERT

Jean WaHL, Kierkegaard. L'Un devant l'Autre. Préf. et notices de Vincent DeleCRoIX, postf. de Frédéric Worms. Paris, Hachette Littératures, 1998. $13 \times 20$, 322 p., bibliogr. (Coup double).

Jean Wahl (1888-1974) fut un lecteur passionné de Søren Kierkegaard, qu'il contribua plus qu'aucun autre commentateur, peut-être, à faire connaître en France à partir des années trente. Les Études kierkegaardiennes (1938) furent une contribution majeure à la découverte et à la compréhension du philosophe danois, reconnu justement comme philosophe, et pas simplement comme écrivain « romantique » ou " mystique ». Les textes réunis ici, articles ou conférences, ne sont pas tous devenus partie intégrante ou annexe des Études; on trouve aussi des textes (notamment sortis 
dans des revues danoises) qui n'avaient jamais été republiés, voire de véritables inédits. On est donc là en présence d'un matériau varié, dont l'écriture s'est échelonnée des années trente aux années soixante, témoignant de la continuité et de la vigueur des réflexions d'un philosophe qui ne fut pas simplement commentateur de Kierkegaard, et dont les thèmes propres transparaissent au fil des articles : Jean Wahl fut l'auteur, entre autres, d'essais sur le Rôle de l'instant dans la philosophie de Descartes (1920), sur Le Parménide de Platon (1926), sur Le Malheur de la conscience dans la philosophie de Hegel (1929), ou encore de Existence humaine et transcendance (1944).

Les textes présentés témoignent donc de la rencontre de deux personnalités philosophiques, mais aussi du contexte d'une époque qui, au nom de Kierkegaard, rattachait immédiatement les notions d'angoisse, de romantisme, de mysticisme, ou les promesses d'un titre tel que Le Journal du séducteur. Cette époque était aussi celle de l'essor des philosophies de l'existence (que Wahl ne réduit pas à l'existentialisme), et l'on ne s'étonnera pas de voir souvent cités les noms de Martin Heidegger et de Karl Jaspers. Le style de l'écriture et du commentaire pourra surprendre par ses aspects impressionnistes, par l'abondance de formules très ou peut-être trop synthétiques, par une pratique parfois désinvolte de la citation. Il faut d'ailleurs préciser que Wahl lisait Kierkegaard dans la traduction allemande et non dans l'original danois.

A-t-on nommé là des défauts rédhibitoires? Certes non : on a simplement défini le cadre dans lequel peut se comprendre et s'apprécier l'apport de Wahl à la lecture de Kierkegaard et, plus généralement, à la réflexion philosophique. Ce qui frappera d'ailleurs le lecteur de ces pages est la clairvoyance avec laquelle Wahl évite de cautionner certains clichés qui se répandaient alors déjà sur Kierkegaard : il rappelle que Le Journal du séducteur est une partie de L'Alternative et qu'il n'avait pas à être publié séparément; il ne réduit pas le rapport de Kierkegaard à Hegel à une opposition stérile, car il reconnaît le poids d'un héritage et le caractère dialectique de cette relation. Concernant le romantisme de Kierkegaard, il prend bien soin de montrer en quoi, si romantisme il y a, c'est chez le philosophe danois sur le fond d'une polémique sans concession avec tous ceux qui, à la vie réelle, préfèrent les possibilités de la rêverie.

On trouvera par ailleurs dans ces pages des analyses alertes sur quelques notionsclés de la pensée kierkegaardienne : le paradoxe, l'angoisse, l'existence, l'individu, qui sont restituées dans une véritable subtilité dialectique, par laquelle Wahl évite les simplismes, même quand il en semble très proche. Une de ses grandes forces est certainement de ne pas prétendre à un décryptage total ou systématique de Kierkegaard : s'il parvient à convaincre de la rigueur conceptuelle de cet auteur, c'est en restituant la vie, le devenir qui fondent les catégories existentielles et les placent dans un rapport polémique à la pensée objective. Pour autant, on n'assiste pas à une dérive subjectiviste : l'individualité kierkegaardienne n'est pas enfant du caprice, elle est redevable à des niveaux différents de Socrate et du Christ, et soucieuse de réalité. Dans cette perspective, la concision et la souplesse de Jean Wahl font merveille pour suggérer l'importance du devenir, de l'incertitude ou de la communication indirecte.

En fait, Kierkegaard est rarement le seul protagoniste de ces articles et conférences : outre Hegel, les compagnons littéraires (Dostoïevsky) et surtout philo- 
sophiques ne manquent pas. Une étude entière est consacrée à « Heidegger et Kierkegaard », qui déborde rapidement le cadre des références explicites du premier au second pour s'épanouir dans une libre confrontation très caractéristique du style de Wahl : le nivellement, l'anonymat, le on, l'angoisse, le temps ou la chute sont des thèmes qui, par-delà l'entrelacement des pensées, vont permettre de rechercher ce qu'a de plus propre la philosophie heideggérienne. De même, la démarche de Kierkegaard vient éclairer celle de Karl Jaspers, et donner les outils d'une étude d'ensemble sur « Les philosophies de l'existence et le renouvellement de la métaphysique ". Le propos est toujours ample, la philosophie généreuse, prompte à établir par-delà les époques et les doctrines la certitude d'une contemporanéité qui est justement l'une des notions kierkegaardiennes étudiées.

Certes, le propos est daté, historiquement situé, et le Kierkegaard qui apparaît dans ces pages n'est certainement plus tout à fait celui que les recherches actuelles tentent d'éclairer : lu sous l'éclairage des philosophies de l'existence, sans référence très consistante au contexte de ses lectures et polémiques danoises, il n'est pas très clairement situé dans son rapport à la mystique. On ne cherchera donc pas là une introduction idéale à Kierkegaard, ni une mise au point sur les travaux les plus récents. Mais on ne se trouve pas non plus en face de textes totalement obsolètes : il s'agit d'une pensée vivante, à la fois rigoureuse et subjective, dont l'intérêt porté à Kierkegaard contient non seulement un hommage mais aussi les éléments d'une réflexion philosophique sur les relations du temporel et de l'éternel, sur l'instant, sur l'existence, la subjectivité et la transcendance. L'intérêt historique de ces textes est réel, mais il ne fait pas oublier qu'il y a là des moments de philosophie, de pensée libre. L'ensemble est accompagné d'une préface et de notices très utiles de Vincent Delecroix, ainsi que d'une postface de Frédéric Worms. Il permet de garder à l'esprit «Ce que nous devons à Kierkegaard", pour reprendre le titre du dernier texte, mais aussi ce que nous devons à Wahl.

Joël JANIAUD

The Cambridge Companion to Nietzsche. Ed. by Bernd Magnus and Kathleen M. HigGins. Cambridge, Cambridge University Press, 1996. 15,2 × 22,8, IX403 p., bibliogr., index (Cambridge Companions).

Cet ouvrage se présente comme un aperçu des études nietzschéennes dans le monde, destiné expressément selon les orientations de la collection dans laquelle il s'insère, à faciliter l'accès aux grands auteurs pour les étudiants et les nonspécialistes. Ce n'est donc pas un travail réservé, ni tourné vers le passé en tant que tel. La question à laquelle on tente de répondre, se veut résolument actuelle, ou ce qui revient au même, si l'on étudie ici Nietzsche et son influence, ce n'est pas dans une perspective d'érudition autosuffisante, mais visiblement avec le souci constant de décider de l'utilité réelle, ou simplement possible, de sa philosophie pour aujourd'hui, et même peut-être aussi pour demain.

De là le style, toujours accessible, et l'orientation de tout l'ouvrage. L'introduction souligne d'emblée les problèmes liés à la perspective adoptée : l'influence de 
Nietzsche, pour incontestable qu'elle soit, n'en est pas moins très ambiguë. Ce constat préliminaire oblige de rappeler aussitôt la responsabilité première des concepts nietzschéens eux-mêmes dans cet état de fait irritant : il est vrai que plus ces derniers ont d'importance dans ses auvres et plus ils apparaissent insaisissables à force d'être self-consuming, sortes de serpents glissants qui se dévorent la queue et insinuent ainsi subrepticement l'incertitude de leur propre rejet. Ainsi se trouve épinglée avec prudence la thèse centralement instable du perspectivisme. Presque tous les articles y reviendront obstinément et buteront en toute loyauté sur le mur de sa question : que faut-il faire aujourd'hui du perspectivisme nietzschéen, s'il n'est lui-même qu'une perspective parmi les autres? Rétrospectivement, la même difficulté n'éclaire-t-elle pas le fait qu'on ait pu à ce point plier et ployer la pensée de Nietzsche un peu dans tous les sens?

Tout le présent ouvrage se charpente en conséquence sur ces constatations fondatrices. Après une première partie qui présente de façon très pédagogique les concepts principaux de la philosophie de Nietzsche dans leur ordre chronologique d'apparition, trois nouvelles parties servent à regrouper les articles dans des perspectives complémentaires : d'abord pour tenter de comprendre quelle est la véritable teneur de cette pensée vivante et vécue au travers même des récupérations plus ou moins fallacieuses auxquelles elle a donné lieu; puis pour étudier son impact jusqu'à nous, à la fois dans le temps (du prémodernisme à la modernité, ou même à la post-modernité), et enfin dans l'espace, en partant de l'Allemagne, pour traverser surtout la France, et s'étendre jusqu'aux confins plus inattendus de l'Asie et de l'Extrême-Orient.

Dans la première partie, comme dans toute initiation, Bernd Magnus et Kathleen M. Higgins fournissent donc les clés indispensables de la lecture et de la compréhension.

Le sens du message, au travers de ses déformations, fait l'objet de l'enquête de la seconde partie. R. J. Hollingdale pose la question de la « légendification » de la vie même du philosophe resté selon lui trop célèbre pour son errance et sa solitude, précisément exagérées par la renommée. Jörg Salaquarda cherche à expliquer le revirement de Nietzsche vis-à-vis du christianisme de son éducation, par les idées fausses que le philosophe s'en fit. Tracy B. Strong soutient que si la pensée de Nietzsche a pu être récupérée par tous les bords politiques, c'est d'abord qu'elle était foncièrement apolitique. Ou alors il faudrait rappeler que, pour lui, la seule politique souhaitable était celle de la polis, comme monde collectif unique et sans but mis en auvre dans la tragédie attique, qui déclina avec elle et fut remplacée par une politique tendant à un but idéal tout au long d'une histoire conduisant à sa fin, comme l'épopée de type aristotélicien.

Après ces mises au point, la troisième partie s'attelle à la question de la valeur actuelle de cette pensée : quelle place peut-elle, doit-elle occuper dans la modernité? Richard Schacht tente d'abord de définir l'originalité de cet inédit mode de pensée : philosophie expérimentale, ouverte, attachée à l'étude de cas concrets et incarnés, usant du perspectivisme non pas comme d'un inhibant relativisme gnoséologique, mais comme d'un moyen d'enrichir sa connaissance par la multiplication des points de vue sur un même objet. Tout cela explique mieux à la fois la finesse et la luxuriance de l'écriture de Nietzsche, et l'incompréhension parfois dédaigneuse dont elle est encore victime chez les philosophes de métier. Robert C. Solomon veut 
cristalliser cette même originalité, et résout encore cette toujours identique difficulté : Nietzsche, contre l'avis des philosophes, use et abuse de l'argument ad hominem, qui fait effectivement partie intégrante de son perspectivisme comme lien indétachable entre la pensée et la personnalité du penseur, et prépare ainsi le retour du bâton avec lequel il a frappé; dans et par sa folie, son æuvre sera à son tour menacée de l'intérieur. D'où la question bilan : Nietzsche est-il encore moderne ? Alexander Nehamas souligne une fois de plus son ambiguité : tantôt le philosophe rejette la tradition, tantôt il s'y arc-boute; en même temps, il dénonce la croyance naïve au progrès et la poursuite d'un but, et il veut encore des buts. L'esthétisme fournit la clé de son perspectivisme et de ses ambivalences, et le modèle de son style finalement post-moderne. Dans une optique parallèle, Robert B. Pippin entreprend de répondre à Jürgen Habermas qui voyait en Nietzsche un rejet de la modernité au profit d'un modèle prémoderne par l'ambiguïté même du philosophe qui triomphe de cette réduction, notamment sur le point fondamental de son attitude ambivalente vis-à-vis de l'optimisme moderne de la connaissance.

La quatrième partie traverse les espaces de l'influence nietzschéenne. Ernst Behler pose des jalons éclairants pour mieux suivre ce parcours étonnant : d'abord, une distinction entre une influence plutôt sur la littérature avant la Deuxième Guerre mondiale et, ensuite, plutôt sur la philosophie. Ce qui mériterait d'être systématiquement rappelé dans les éditions, c'est que les idées de Nietzsche sont toujours vues à travers les préjugés et les préoccupations d'un temps : ainsi pour Martin Heidegger dans les années trente, Nietzsche évoque le déclin, pour Walter Kaufmann après guerre cette même pensée apparaît définitivement apolitique. Alan D. Schrift dresse le catalogue des héritiers français du «new and French Nietzsche », tantôt commentateurs érudits, tantôt écrivains et créateurs. Enfin, Graham Parkes nous ouvre des horizons peu connus en reliant trois problèmes complémentaires : le peu que Nietzsche doit à la pensée orientale dans l'élaboration de sa propre pensée; inversement, l'influence très importante (ce qui est une découverte, en tout cas pour moi) de Nietzsche sur les penseurs orientaux, et surtout japonais; enfin, une comparaison plus distanciée entre les idées du philosophe allemand et celles de la tradition chinoise.

Le panorama proposé par ce livre apparaît utile et assez complet. Toutefois, en tant qu'ouvrage d'initiation, cela appelle quelques réserves. On notera d'abord un défaut, trop universitaire, de méthode : pour une première approche, on discute trop souvent des commentateurs, et on ne revient pas assez aux textes de l'auteur luimême; cela tourne un peu à la conversation de sérail, alors qu'il y a tant à découvrir dans l'original lui-même. Quelques lacunes de contenu, parfois surprenantes, sont à regretter : pour ce qui est du retentissement de l'œuvre, n'est-il pas étonnant de ne presque rien trouver concernant la période nazie? Ne convient-il pas au contraire de prévenir loyalement les jeunes lecteurs? Philosophiquement, le lien serré entre la vie et la philosophie du penseur est souvent évoqué, mais sa biographie, d'une manière incompréhensible, ne se présente nulle part. Enfin, et pour ne plus séparer contenu et méthode, comment faut-il prendre le silence obstiné sur les relations pourtant déterminantes à Richard Wagner, ainsi que l'impasse à peu près totale sur la place, en fait décisive, de l'art et de la musique dans l'élaboration et la présentation de cette pensée unique? C'est d'autant plus dommage que cela ferme la compréhension de certains points forts comme la volonté de puissance et la volonté 
de connaissance, et ouvre au contraire la porte à une crispation excessive sur le problème du perspectivisme entendu comme relativité psychosociologisante des points de vue. En revanche, le véritable fondement généalogique et typologique du perspectivisme est peu signalé et encore moins vraiment utilisé pour unifier, comme il le devrait, les aspects les plus divers de la pensée du philosophe allemand, et le laver, enfin, du soupçon finalement déplacé, de ne s'être jamais "self-consumé ». En un mot, cet ouvrage a le défaut de sa qualité, et risque d'être plus utile aux spécialistes qu'aux débutants.

Dominique Catteau

Ali Benmakhlouf, Bertrand Russell. L'atomisme logique. Paris, Presses universitaires de France, 1996. 11,5 × 17,5, 128 p., bibliogr. (Philosophies, 70).

Ali Benmakhlouf entend nous présenter la philosophie de Bertrand Russell sous l'angle de l'atomisme logique qu'il définit très clairement comme : « [...] la considération des données des sens et des images comme éléments discrets, privés et non inférés, des atomes en somme »(p.6). En fait, il ne s'agit pas seulement d'un simple commentaire linéaire des conférences de 1918-1919 sur la philosophie de l'atomisme logique. Même si l'ordre de l'ouvrage est calqué sur celui des conférences et que les deuxième et troisième parties les commentent explicitement, l'ouvrage nous apprend beaucoup plus dans la mesure où il étudie de façon générale l'atomisme logique, son contenu mais aussi ses présupposés et ses conséquences.

Trois parties traitent successivement de l'atomisme logique comme théorie de la connaissance, comme philosophie des faits et comme théorie de la réalité et de la fiction.

Dans l'introduction, l'auteur situe Russell et sa perspective philosophique par rapport à ses contemporains, c'est-à-dire Wittgenstein, Frege, Péano, Einstein, mais aussi Dewey, Carnap et Hempel. Il le place dans la tradition des empiristes anglais (Locke, Berkeley, Hume) et dessine sommairement son évolution philosophique, Russell ayant d'abord été idéaliste, puis réaliste « luxuriant », puis réaliste « sobre ».

La première partie de l'ouvrage est consacrée à l'atomisme logique comme théorie de la connaissance. Il s'agit d'un "réalisme analytique " dans la mesure où il s'intéresse aux données des sens - et non aux objets physiques inférés - qu'il consídère comme les constituants simples de la réalité. Mais cela même suppose une théorie de la compréhension : comme ces faits ne peuvent être que constatés, on ne peut connaître que directement ou par " accointance ». L'auteur, qui ne manque jamais de signaler que la pensée de Russell a connu des évolutions, explique comment ce dernier est passé d'un dualisme entre sense-data et sensation, à un monisme proche du monisme neutre. Une telle théorie de la connaissance induit forcément une conception particulière du sujet qui se trouve réduit à un point de vue irréductible, et ce d'autant plus que bien que les perceptions et les sensations soient privées, elles restent le seul point de départ possible pour notre connaissance du monde extérieur. 
La seconde partie concerne plus directement les conférences de 1918-1919. Il y est question des faits et des énoncés qui leur correspondent, la théorie de la vérité comme correspondance, soutenue par Russell, différant beaucoup de celles de la vérité comme cohérence ou comme vérification. La nécessité d'une théorie du symbolisme est mise en évidence, afin de dégager les différents sens de "signification " et de mettre en place ce que Russell appelle une « ambiguité systématique », c'est-àdire une possibilité pour le même mot de signifier plusieurs choses pour éviter les confusions. Les faits étant toujours complexes, seule la connaissance directe de leurs composants permet de comprendre les propositions qui leur correspondent. Le recours à un langage logiquement parfait n'est qu'une hypothèse au sujet de la possibilité d'analyser les symboles à la place des faits auxquels ils correspondent et, dans l'idéal, on ne peut nommer que ce dont on a une connaissance directe; le reste ne peut être que décrit, étant entendu qu'une description ne signifie pas comme signifie un nom propre.

La dernière partie étudie le rapport entre la réalité et la fiction. Benmakhlouf aborde la question de l'expression de la généralité et de l'existence et de leur signification; un des grands mérites de Russell est, en effet, d'avoir montré que l'existence n'est pas une propriété des individus mais des fonctions propositionnelles signifiant que ce qu'elles affirment est parfois le cas. Il s'intéresse ensuite à l'inventaire des faits (atomiques, de croyance, généraux, d'existence) et montre que les théories des descriptions définies, des types et des fictions logiques sont des théories réalistes qui empêchent la multiplication indue des entités : à chaque fois, il s'agit de faire agir le rasoir d'Occam afin de faire disparaître toutes entités ou tout sujet métaphysiques et de les remplacer par des constructions logiques fictives capables de jouer le même rôle. Finalement, Russell n'admet que l'existence des faits et de ces indéfinissables que sont les particuliers, les qualités et les relations : il est constructiviste tout en restant réaliste. Certes, sa volonté de résoudre certains problèmes philosophiques à l'aide de moyens logiques le rapproche des positivistes logiques mais, contrairement à eux, il prend toujours en compte le problème psychologique de la compréhension, car il estime qu'une théorie de la signification n'a pas de sens sans théorie de la compréhension.

La conclusion réaffirme donc le réalisme de Russell en montrant que l'analyse n'est pas falsificatrice ni même réductrice et que l'atomisme logique est une véritable théorie de la connaissance qui prend en compte et l'aspect logique et l'aspect psychologique.

Cet ouvrage a le mérite d'insister sur l'intérêt philosophique de la pensée de Russell et de le présenter de façon synthétique : «Russell défend l'idée d'une grammaire philosophique comme réponse aux problèmes traditionnels de la métaphysique : la critique de la substance à partir du jugement relationnel, la mise en place de la théorie des types pour échapper aux paradoxes de l'auto-référence, la refonte de la notion d'existence à partir de son acception mathématique, la mise en évidence des pseudo-noms propres, la théorie des particuliers particuliers et des particuliers généraux. Mais on peut aborder tous ces aspects à partir d'une notion (celle de fiction logique) et d'une règle (celle du rasoir d'Occam)» (p. 110).

Benmakhlouf montre bien comment la pensée de Russell évolue et comment, à chaque étape, il se situe par rapport aux pensées de ses contemporains. Il insiste à plusieurs reprises sur la richesse, la complexité et l'originalité de cette philosophie 
en montrant qu'elle doit être considérée comme une véritable théorie de la connaissance faisant une place aussi bien à la logique qu'à la psychologie. En bref, il a le mérite de nous persuader, à partir de l'étude d'un texte en particulier, que Russell est un véritable philosophe digne d'être étudié dans le détail.

Frédérique DEsForges

Jocelyn BenoIst, Phénoménologie, sémantique, ontologie. Husserl et la tradition logique autrichienne. Paris, Presses universitaires de France, 1997. $15 \times 21,5$, 313 p., index (Épiméthée).

Jocelyn Benoist fonde son étude "Husserl et la tradition logique autrichienne » sur l'idée, présentée d'emblée, qu'on ne saurait accuser Husserl de céder au mythe de la signification : le sens n'est pas une chose, mais est produit dans des actes, qui sont des actes de visée. L'idéalité des significations, irréelle en tant qu'idéale, n'est que l'unité spécifique des vécus identiques, présente à même le vécu, et n'est pas une chose. L'identité du signifier elle-même n'est pas une chose, elle est dans l'acte remplissant, qui la spécifie et la détermine : c'est le sens réalisé, actualisé. Ni son idéalité ni son identité ne suffisent à faire du sens une chose.

Qu'est-ce que le sens s'il n'est pas ce mythe ? Pour le savoir, il faut en passer par le remaniement que Husserl fait subir à la notion kantienne d'analyticité, et par Bolzano, qui lui donne les moyens de ce remaniement. Bolzano découvre la signification moderne de l'analyticité comme opération formelle de substituabilité, qui permet de réduire la proposition à un ensemble de constantes logiques, et de maintenir la valeur de vérité quelles que soient les valeurs des variables. Husserl généralise cette notion, ne la limite pas à une région ou à un contenu déjà donné qui figurerait dans une proposition identique, mais montre que c'est le signifier lui-même qui se formalise. Ce n'est pas seulement tout contenu qui est formalisé, mais le signifier en tant que tel est puissance de formalisation. Cette puissance de formalisation s'oppose à la sémantique, qui part toujours de tel ou tel contenu pour en donner le sens : le sens est donc au-delà de la sémantique.

Cette puissance de formalisation du sens se découvre comme syntaxe : à la suite de Franz Brentano et de sa théorie du jugement comme position, à la suite de Marty et de sa fondation des propositions impersonnelles et des propositions prédicatives dans la positionnalité du jugement, on découvre qu'il y a, au-delà du langage et des significations des contenus de la proposition, une articulation originaire de la signification, qui est présente dans le langage, comme sa logique, mais s'y dissimule sous l'apparence linguistique - une sorte de grammaire profonde. Les propositions impersonnelles - sans sujet - apparaissent tout de même avec un sujet, bien que leur impersonnalité soit un caractère syntaxique irréductible.

Le catégorial est le nom pour cette " plasticité de la forme » comme puissance du signifier. La relation garde son sens quelles que soient les valeurs de ce qu'elle unit. Il suffit de comparer un tout sensible et les données formelles d'un ensemble pour avoir un autre exemple de formalisation. L'abstraction formalisante ne retire aucune 
partie à ce dont elle abstrait, mais en exhibe la forme. Nous éprouvons cette formalisation à l'œuvre quand nous parlons, car les catégories de la signification sont les contraintes inhérentes au fait d'employer le langage. La logique des actes signitifs cherche à formaliser l'origine sémantique des catégories, sans partir des catégories comme contenus dans la langue.

L'auteur montre ensuite la priorité du sémantique sur l'ontologique : la nominalisation, qui est une opération sémantique, a une prise sur l'ontologique, en faisant ressortir comme signification indépendante un moment ontologiquement dépendant, par exemple le rouge. L'intuition est travaillée par le signitif quand elle exhibe les moments ontologiques, et les catégories de la signification sont premières en ce sens.

La troisième Recherche logique sur le tout et les parties montre que l'ontologie elle-même est atteinte par cette puissance de formalisation propre au signifier qui la fonde en un certain sens. Les relations de dépendance et d'indépendance ne lient pas des représentations, elles sont les objets eux-mêmes, qui exercent ainsi leur contrainte différenciée sur la pensée ou la perception. Ainsi se trouve donné ce que l'auteur appelle un plan d'immanence : abstraction et concrétion sont des propriétés ontologiques de ce plan d'immanence, et non pas psychologiques. On ne peut plus se contenter de la simple forme d'un objet, double ontologique de ce qu'est le sujet dans le jugement prédicatif, mais l'objet n'est plus que sa spécification et sa détermination selon l'a priori matériel.

La pensée est déterminée par les objets qu'elle pense. L'élargissement du sens de l'objectivité doit aller de pair avec la découverte de l'autonomie de la signification, ce que l'auteur montre en comparant Husserl à Alexius Meinong qui élargit le sens de l'objectivité mais en reste à une ontologie représentationnaliste de l'objet comme ce qu'il y a en dehors et qu'on doit se représenter. Meinong prétend se passer de la signification comme troisième terme, si bien que le catalogue des objets préexiste à leur emploi; pour Husserl, l'emploi et les objets sont coextensifs, et l'élargissement de l'objectivité se double d'un nouveau sens, fondé sur le signitif, d'objet. C'est à l'aune de cette puissance de formalisation du sens comme plan d'immanence qu'il faut reconsidérer les concepts fondamentaux de la phénoménologie dans les Recherches logiques, ainsi que leur caractère soi-disant métaphysique.

En tant que théorie de la connaissance, la phénoménologie est logique pure, et non pas théorie de la connaissance au sens kantien de fondation de l'effectivité. En effet, la science mathématique par exemple ne concerne pas l'effectivité, la réalité naturelle, alors que la métaphysique ne porte que sur ces questions de réalité naturelle et d'effectivité. D'où la nécessité d'une théorie de la science qui soit interrogation sur la science au sens de science qui excède la métaphysique, qui n'est pas au-delà, mais hors de la métaphysique.

C'est pour adopter la neutralité exigée par cette théorie de la science qu'il faut adopter la méthode phénoménologique - réduction et description. Mais pourquoi alors caractériser la phénoménologie comme « psychologie descriptive » ? C'est que Husserl oppose une psychologie descriptive, non théorétique, à une psychologie génétique, atteignant par là un positivisme de la description, qui cherche à appréhender des phénomènes, et non pas à fonder le psychique.

L'ambiguité constante entre logique et psychologie se résout dans une primauté du logique, et Husserl ne se place sur le terrain de la psychologie que juste assez 
pour ontologiser la conscience. Husserl ne réintroduit pas, par exemple, un gouffre entre sensation et objet, intériorité et extériorité, mais en se tenant à l'intérieur du plan d'immanence, montre que sensation et objet sont deux manières de se donner, d'apparaitre qu'il faut consigner descriptivement. Demeurer dans le plan d'immanence, c'est comprendre que le phénomène est l'apparition d'un objet accompagnée de l'épreuve qui lui est propre comme apparaître, sentir originaire. Le moi n'est ainsi qu'une sphère pure de l'apparaître, l'ensemble des données phénoménologiques, la conjugaison immanente des vécus comme unité du flux.

L'auteur montre ainsi qu'il faut, chaque fois que Husserl emploie l'expression " perception interne », comprendre "perception adéquate ", car l'adéquation signifie la donnée sans interprétation ni représentation de la chose elle-même, l'absence de distance entre le vécu et ce qui est vécu. Il n'y a même pas intériorité de l'adéquat au vécu, car l'immanence est première, le vécu second. La conscience est laisser être de l'apparaître, et non pas savoir de soi. L'intériorité n'est pas adéquate, c'est l'adéquation qui est intériorité. Et même l'absence d'évidence de ce qui est donné fait partie de l'évidence. C'est sur le fond d'une structure ontologique formelle et matérielle que l'intentionnalité se déploie comme usage de cette structure. Faire sens, c'est se donner à éprouver les contraintes de l'apparaître. Le sens n'apparaît pas, et dans ce non-apparaître il se sent, mais l'objet apparaît et se donne à connaître en lui.

\section{À partir de l'ouvrage de Benoist, trois questions peuvent se poser :}

L'auteur montre combien central est le concept de formalisation dans la pensée husserlienne, mais la formalisation n'est pas seulement la puissance de se formaliser du sens, elle est aussi et surtout pour Husserl un moyen d'appréhender le sens et la signification. Qu'est-ce positivement que cette formalisation si c'est une formalisation phénoménologique, c'est-à-dire une méthode phénoménologique pour faire du sens un objet de connaissance sans céder au mythe de la signification? Si la formalisation est une opération méthodique proprement phénoménologique visant à saisir la puissance de formalisation du sens, quelle est-elle? La réponse est peut-être à chercher du côté de la variation : mettre en variables, c'est d'abord faire varier, et la pièce méthodique du Husserl plus tardif est la variation - dont il faut chercher les linéaments dans les Recherches logiques, et en considérant que, sur ce point au moins, les Idées I et $I I I$ marquent un progrès important. Qu'on la comprenne comme variation eidétique ou autrement, elle joue à tous les niveaux où il s'agit de connaître le sens - sans oublier que la première parution de Husserl porte sur le calcul des variations. Car l'auteur ne donne d'autre sens à cette idée de formalisation que mathématique ou logique, mais la formalisation en mathématique ou en logique, c'est précisément ce qu'il ne nous est pas donné de voir, ce qui n'apparaît que sous la forme d'une forme. Husserl ne fait ni une mathématique ni une logique mais s'interroge sur la notion de sens et de signification, et de cette manière tente de mettre à jour le concept original et non métaphysique de formalisation tel qu'il est à l'œuvre implicitement dans les mathématiques ou la logique formelle.

Décrire comme Husserl son projet comme celui d'une « théorie de la connaissance ", n'est-ce pas s'entendre sur un certain sens de connaissance, pris sous un 
regard théorétique, ou du moins présupposer le fait de la connaissance elle-même? La cognoscibilité en droit des objets, qui fonde le fait que la signification vise des objets, n'atteste-t-elle pas cette présupposition? Autrement dit, une véritable phénoménologie de la connaissance ne doit-elle pas laisser apparaître la connaissance, à partir de la non-connaissance, au lieu de partir, comme les néo-kantiens, du fait de la connaissance? Ne restreint-on pas la puissance du sens si on présuppose cette connaissance, si la connaissance n'a pas à apparaître dans la lumière du sens luimême? Une phénoménologie de la connaissance ne doit-elle pas s'entendre en un sens non théorétique?

Enfin, la découverte du signifier permet-elle à Husserl de dépasser autrement qu'en droit la puissance de fait, logée dans l'apparence linguistique, du jugement prédicatif? Rien n'est moins sûr, et des auteurs analytiques comme Tugendhat l'ont montré. Le sens est rabattu sur la seule forme syntaxique que peut prendre la connaissance : le jugement prédicatif. La sixième Recherche logique confirme en maints endroits cette appréciation.

Il y a trois manières d'approcher les Recherches logiques : les lire dans l'économie de l'œuvre husserlienne et à la lumière de son tournant transcendantal, qu'elles prépareraient; contrarier la pente naturelle de la téléologie herméneutique en œuvrant à une archéologie des Recherches logiques, en la mettant en rapport avec le contexte logique où elle émerge; enfin, tenter de voir ce qui, dans les Recherches logiques, est le lieu de l'explication, au sujet de la phénoménologie, entre Heidegger et Husserl. Le livre de Benoist, clair et passionnant à lire, nous semble exercer à plein cette lecture archéologique qui contrarie si bien la tendance naturelle de la téléologie qu'elle libère l'œuvre pour cette troisième lecture qui engage l'essence de la phénoménologie.

Philippe QUESNE

Jacques Merleau-Ponty, Einstein. Paris, Flammarion, 1993. 13,5 × 22, 299 p., bibliogr., chronol. (Figures de la science).

Voici un ouvrage qui sans doute restera, malgré d'inévitables lacunes dans l'état actuel de la documentation, le meilleur travail en langue française portant sur l'ensemble de la vie et de l'œuvre de l'illustre physicien.

Albert Einstein est né en mars 1879 à Ulm, dans une famille de commerçants juifs en literie; poussé par un oncle entreprenant avec lequel il s'associa, son père fonda en 1880 une affaire de distribution d'eau et de gaz à Munich, puis un peu plus tard une usine de matériel électrique. La sceur d'Einstein, Maria, naquit en 1881. On doit relever surtout, de sa prime enfance, l'émerveillement qui s'empara de lui à la vue d'une boussole et dont il garda le souvenir jusque dans sa vieillesse. $\grave{A}$ six ans, il entra à l'école publique, y obtint de bons résultats, en calcul notamment. Il reçut de sa mère, pianiste, une excellente éducation musicale qui devait lui apporter sa vie 
durant plus qu'un délassement : des joies profondes, liées à de très fidèles amitiés. Élève du Gymnasium (lycée) de Munich de 1888 à 1895, il y fit de très bonnes études, en mathématiques surtout, mais déconcerta ses professeurs par un certain détachement teinté peut-être d'ironie critique à l'égard des autorités. Lorsque ses parents décidèrent de s'expatrier en Italie, le jeune homme fut mis en pension dans une famille suisse de langue allemande, les Winteler, où il trouva à se réjouir d'une ambiance « démocratique ", bien différente de celle de son pays natal. Il se présenta au concours d'entrée au Polytechnikum de Zurich, ne réussit pas, mais obtint l'autorisation de suivre le cours de physique de Heinrich Weber tout en préparant la " maturité » suisse (équivalent du baccalauréat). En 1896, il entre au Polytechnikum et $y$ poursuit une formation d'enseignant en mathématiques et physique pendant quatre ans. Alors apatride, il devra attendre 1901 pour acquérir la nationalité suisse. Au Polytechnikum, il fait la rencontre de Mileva Maric, une étudiante serbe très cultivée, dont on ignore encore si elle contribua activement aux recherches que commençait Einstein, mais qui était en tout cas de taille à suivre et à comprendre ses travaux. Toujours est-il que les deux jeunes gens tombèrent amoureux l'un de l'autre, un premier enfant naîtra, hors mariage, en février 1902, une fille nommée Lieserl. Ils se marièrent en janvier 1903, Einstein avait près de 24 ans, Mileva (1875-1948) en avait 28; ils eurent un premier fils, Hans Albert (1904-1973) (futur professeur à Berkeley, CA); le second, Édouard (1910-1965) eut une plus triste destinée et mourut en hôpital psychiatrique à Zurich. Recommandé par le père d'un condisciple, Einstein obtint un emploi d'expert à l'office des Brevets de Berne, travail absorbant dont il s'acquitta avec maîtrise et efficacité, à la satisfaction du directeur de l'office, de 1902 à juillet 1909.

1909 marque aussi son accession assez discrète à la carrière universitaire comme professeur associé à l'université de Zurich. Mais c'est plus tôt, en 1905, que s'affirme une méthode plus serrée et que le physicien discipline mieux sa pensée, produisant notamment deux chefs-d'œuvre, le mémoire sur les quanta de lumière (en mars) et le mémoire sur l'électrodynamique des corps en mouvement (en juin), complété (en septembre) par celui sur l'inertie de l'énergie. Dès lors se nouent de solides amitiés entre spécialistes renommés, tels que Max Planck, son assistant Max von Laue, Paul Langevin à Paris, Hendrik Lorentz en Hollande, Ernest Rutherford, Arthur Eddington en Grande-Bretagne d'une part, et de l'autre, le physicien naguère encore inconnu, qui accède ainsi à la célébrité. Hermann Minkowski, ancien professeur au « Poly » de Zurich, devenu professeur à l'illustre université de Göttingen, où enseignaient les mathématiciens les plus en vue, apporta une contribution décisive à la théorie de la relativité en inventant la notion unitaire de l'espace-temps.

En octobre 1909, Einstein prend ses fonctions de professeur associé à l'université de Zurich. En octobre 1911, a lieu à Bruxelles le premier congrès Solvay (du nom d'un riche industriel passionné de physique); Einstein y fut invité comme la plupart des sommités en la matière, Marie Curie, Langevin, Planck, etc., et il y prononça une conférence sur les chaleurs spécifiques; en mars, il s'était installé à Prague pour y occuper la chaire de physique théorique. Ce fut pendant cette courte période qu'il connut les débuts d'une gloire véritable, que l'après-guerre devait encore amplifier. En 1912, il est nommé professeur au «Poly » et revient à Zurich. Toutes ces années abondent en travaux importants dont beaucoup visent à la clarification logique et heuristique de l'acquis. En février 1919, il divorce d'avec Mileva; les enfants de ce 
premier lit restent en Suisse avec leur mère; en juin, il épouse sa cousine divorcée Elsa et la prend en charge avec ses deux filles. Il s'intéresse en même temps au pacifisme et au sionisme (sans croire au mythe du peuple élu). Dès 1913, Planck et un collègue, Walther Nernst, avaient fait le voyage de Zurich pour proposer à Einstein un ensemble d'avantages impossibles à refuser : outre un traitement très important, sa venue à Berlin ne s'accompagnerait d'aucune obligation d'enseignement, il aurait un siège à l'Académie prussienne des sciences, une chaire à l'université de Berlin et, enfin, la direction d'un futur institut destiné à trouver des fonds pour la recherche. Einstein fut élu à l'Académie presque à l'unanimité en juillet 1913; mais la guerre arrive. Bien que malade en 1917, son activité ne se ralentit pas: 1916, approfondissement de la théorie quantique, mémoire sur le rayonnement; 1917 , son mémoire sur la cosmologie inaugure la phase moderne et scientifique de cette discipline. En mai, l'éclipse de Soleil permet à Eddington de confirmer les prévisions relativistes, ce dont Lorentz informe Einstein en septembre; la réunion britannique de proclamation officielle des résultats eut lieu le 6 novembre, avec comme effet, malgré quelques refus d'y croire, une énorme sensation de portée internationale, telle que jamais de son vivant n'en avait recueilli aucun homme de science du passé. En octobre 1920, il donne à Leyde une leçon inaugurale sur la relativité : il est dès lors professeur associé à cette université. Bien que la passion de la recherche domine toute sa vie, il ne s'isole pas et multiplie les contacts divers, qu'il réussit à entretenir fidèlement (c'est ainsi qu'il connaît Romain Rolland, en exil en Suisse, Sigmund Freud, Carl Gustav Jung, etc.). Là où le portent ses voyages, il donne de fréquentes conférences, aux États-Unis en 1921, à Paris en 1922, au Japon où il est reçu avec enthousiasme à la fin de l'année, après avoir obtenu le prix Nobel de Physique pour 1921. En 1924, s'ouvre à Potsdam l'institut Einstein; le maître garde la double nationalité suisse-allemande. En 1925, il fait un voyage en Palestine. L'année 1928 est marquée d'un accident cardiaque. Les voyages se poursuivent : en 1929, en Belgique, en 1931 et 1932, aux États-Unis. En octobre 1932, il entre à Princeton, mais ne quitte l'Allemagne qu'en décembre (à la veille de l'accession des nazis au pouvoir). En 1933, il quitte la Belgique pour l'Angleterre, puis part pour les États-Unis (octobre, Princeton). Il est reçu officiellement début 1934 par Roosevelt (il lui écrira en août 1939 sur la bombe atomique et sera admis comme consultant aux recherches en explosifs de la Marine en 1943). En août 1948, Mileva, sa première épouse, meurt en Suisse; en décembre, il subit une opération cardiaque. Il perd sa sœur Maia, paralysée depuis 1946 et recueillie chez lui avec ses enfants, à la suite de leur expulsion d'Italie par les fascistes, en 1951 (il avait déjà perdu sa seconde épouse en 1936 et sa mère en 1920). Il signe son testament en 1950 instituant le dépôt de ses écrits à la Bibliothèque hébraïque de Jérusalem. Il perd en 1955 son ami de jeunesse, connu en Suisse avant la fin du siècle précédent et resté très lié avec lui, Michele Besso. En 1940, il avait obtenu la double nationalité américaine et suisse; en 1952, il avait décliné l'offre de prendre la présidence de l'État d'Israël.

Il mourut dans la nuit du 17 au 18 avril 1955, à Princeton; selon sa volonté, il n'y eut aucune cérémonie, il fut incinéré. Il avait poursuivi ses multiples activités presque jusqu'à la fin.

L'œuvre scientifique et philosophique occupe près des deux tiers de l'ouvrage. 
Publiés avant 1960, deux cent soixante-dix textes scientifiques se laissent classer sous trois rubriques principales :

1) Les contributions à la physique classique, d'abord de 1901 à 1902 (capillarité et forces moléculaires), puis, de 1902 à 1904, la tentative de définition des fondements de la thermodynamique, enfin et surtout, de 1905 à 1908, les mémoires sur le mouvement brownien, remarquables à la fois par le désir de lui donner un statut théorique et de trouver des moyens suffisamment fins et précis pour vérifier à partir de là l'hypothèse admise du mouvement aléatoire des molécules.

2) Les mêmes recherches (théorie moléculaire de la chaleur) mènent, bon gré mal gré, à une voie radicalement nouvelle, l'invention de la mécanique quantique : après Maxwell et Boltzmann qui menaient à une théorie médiatrice entre les lois de la mécanique et celles de l'électrodynamique, à savoir la mécanique statistique, il convenait d'étudier, ce qui fut l'œuvre de Planck, d'Einstein et de Niels Bohr, le problème du rayonnement du corps noir, dont il fallait trouver la loi d'équilibre entre émission et absorption du rayonnement et aussi le moyen d'une intervention sur le corps noir qui permît avec une suffisante approximation de faire des mesures sur son rayonnement interne. Planck était sûr de sa formule discontinuiste, mais ne voyait pas comment la justifier. Einstein montre (règle empirique de Stokes) que tout s'éclaire si absorption et émission se font par quanta proportionnels aux fréquences. En 1906 et 1907, poursuivant son effort théorique, il s'intéresse aux chaleurs spécifiques des solides et rationalise en l'élargissant la conception des échanges d'énergie entre matière et rayonnement; en 1909, au colloque de Salzbourg, il expose comment les fluctuations du rayonnement du corps noir autour de la position d'équilibre respectent une discontinuité en relation avec la théorie relativiste des échanges d'énergie déjà cités; reste énigmatique la dualité onde-corpuscule. Il n'abandonne une présentation quasi sceptique des quanta (premier congrès Solvay) qu'en 1916-1917 et en reprenant la formule exponentielle de la radioactivité (Rutherford, 1900), il pose l'existence du photon; en 1923, Arthur Compton prouve que le quantum de lumière (le photon) possède une quantité de mouvement. En 1924, les travaux de l'indien Satyendranâth Bose, remarqués grâce à Einstein, conduisent celui-ci à préciser la statistique quantique du gaz parfait (règles de Bose-Einstein), puis (congrès Solvay de 1927) à instaurer une critique de la mécanique quantique contre Bohr et de nombreux physiciens; la même année, Bohr expose à Côme sa théorie dite de la complémentarité qui admet parfaitement la dualité ondes-corpuscules et (congrès Solvay de 1930) s'oppose à Einstein en défendant les relations d'incertitude de Werner Heisenberg (fondement d'une mécanique quantique de base statistique). Einstein tente pour sa part de mettre au point divers procédés théoriques, notamment la boîte à photons et l'argument EPR (Einstein-Podolsky-Rosen), mais sans jamais réussir (au cours de la longue période de 1927 à 1935) à tirer de ces prémisses une confirmation expérimentale prouvant la non-complétude des relations d'incertitude (d'ailleurs admises par tous, sur le plan instrumental, Einstein compris) et la présence d'un déterminisme sous-jacent dans la nature physique; à ce déterminisme, il crut jusqu'au bout.

3) La troisième rubrique concerne la relativité, d'abord dans ses limites (mouvement uniforme), puis généralisée. Einstein part, très précocement, des acquis de 
Newton; il semble mettre en question espace, temps et mouvement « absolus », réalisme indispensable au fondement des mesures astronomiques, en tant que leur substrat sensoriel est repérable adéquatement par le Soleil et les astres fixes, tandis que les mouvements planétaires déterminent naturellement une horloge " absolue ". Mais le mouvement de la Terre est-il objet d'expérience? Au niveau de la recherche de la fin du XIX ${ }^{e}$ siècle, ce mouvement n'est plus qu'un mythe, une fiction pour mieux dire. Néanmoins, on se contente partout des lois de la nature physique, parvenues à un extrême raffinement (mécanique séparée de l'électromagnétisme, même après l'échec des expériences soignées d'Albert Michelson, 1881, répétées ensuite avec Edward Morley et reprises enfin par Einstein lui-même. Désireux, à peu près seul, de surmonter une insatisfaction née de l'insuffisance des systèmes classiques, Einstein est le premier à discerner un problème dans la notion de la relativité du mouvement : la vitesse de la lumière dans le vide est un invariant-limite, seul fondement logique et physique unissant avec précision théorique d'abord, vérification expérimentale ensuite, l'unité exacte matière-énergie (1905, mars, juin et septembre, mémoires sur la relativité restreinte; 1907 : relativité générale, première esquisse; 1919 : proclamation officielle à Londres des résultats astronomiques tirés par voie déductive de la théorie relativiste, Einstein est alors âgé de 41 ans). Sur la généralisation de la théorie, il dira dans son Autobiographie de 1949 (citée p. 179) que c'est « la meilleure idée qu'il ait eue de toute sa vie ».

Il faut également ajouter à la liste des créations dans le domaine des théories scientifiques, celle d'une cosmogonie (à partir de la fin de 1916), c'est-à-dire d'une structure et d'une évolution « historique » au sens le plus vaste, applicable à l'univers en sa totalité. Avec l'aide de son ancien condisciple Marcel Grossmann qui montre l'intérêt de la géométrie riemannienne de l'espace, Einstein bâtit au fil des ans - surtout en 1921 et en 1945, à la suite de longues méditations silencieuses et des découvertes de l'astronome Edwin Hubble sur l'expansion de l'univers - sa conception relativiste de la gravitation, sans plus aucune distorsion logique dans la courbure de l'unité espace-temps.

Dès la période de jeunesse, alors étudiant à Zurich, au cours de réunions périodiques de confontation entre amis, Einstein fut attiré par la réflexion philosophique, soit par le caractère même de sa méthode scientifique en physique (procéder par hypothèse pour résoudre les contradictions et ensuite seulement avoir recours à l'expérimentation), soit en fonction des valeurs morales auxquelles il croyait en humaniste convaincu. De ces deux points de vue, il n'a jamais substantiellement varié, préférant à tout autre, quant à lui, le plaisir de rechercher la vérité, comme il sut le faire jusqu'à la fin de sa vie. 
Béatrice Han, L'Ontologie manquée de Michel Foucault. Entre l'historique et le transcendantal. Grenoble, Jérôme Millon, 1998. 13 × 21,5, 328 p. (Krisis).

La quatrième de couverture indique d'emblée l'ambition de cet ouvrage : traiter Michel Foucault en philosophe. On ne s'inquiétera donc pas de savoir si, oui ou non, « dès les premiers mois de l'enfermement les vénériens appartiennent de plein droit à l'Hôpital Général » - ce que contesta vigoureusement l'historien Claude Quétel; ni de pointer tel ou tel anachronisme de l'Histoire de la folie à l'âge classique. Mais, philosophe, Béatrice Han veut lire en philosophe l'œuvre d'un auteur qui se reconnaît «malgré tout philosophe », et en interroger la cohérence.

Remarquant que le «corpus foucaldien est scandé par deux longs silences »entre L'Archéologie du savoir et Surveiller et punir d'une part, et entre le premier et le deuxième volume de l'Histoire de la sexualité, d'autre part - B. Han distingue donc trois périodes auxquelles correspondent les trois sections de son livre : la première est centrée sur la notion d'archéologie, la seconde sur celle de généalogie et la troisième est, pour l'essentiel, consacrée à la question du sujet et de la vérité. Ainsi cet ouvrage nous offre-t-il une relecture critique de l'ensemble de l'œuvre de Michel Foucault.

Comme l'indique explicitement son titre, "La transposition archéologique de la question critique et les apories du thème transcendantal », la première section privilégie la critique par Foucault de la pensée kantienne à laquelle, du reste, il emprunte le terme même d'archéologie. Au cours de cette période, Foucault s'interroge sur les conditions de possibilité du savoir tout en rejetant le transcendantalisme de la Critique de la raison pure. Paradoxalement, il développe alors le projet d'une archéologie qui se donne pour objet de dégager « ce à partir de quoi connaissances et théories ont été possibles » et que Foucault considère comme un « a priori historique " - étrange concept s'il en est, emprunté à Edmund Husserl et à son Origine de la géométrie où il a un sens supra-historique.

B. Han distingue quatre acceptions différentes de l'a priori historique selon qu'il s'agit d'un a priori antérieur à toute perception dans la Naissance de la clinique; d'un rapport implicite entre les mots et les choses, entre le langage et l'être, qui postule un nominalisme radical; des codes fondamentaux d'une culture; ou, enfin, de la condition purement empirique, selon L'Archéologie du savoir, de réalité pour des énoncés. Â chaque fois, l'auteur souligne les difficultés que suscite ce concept au sein même de l'œuvre, et s'interroge ensuite sur la possibilité de son enracinement - lorsque la Naissance de la clinique tente une archéologie du regard médical dans une phénoménologie de la perception au sens de Maurice Merleau-Ponty, ou encore dans l'ontologie heideggérienne : la différence ontologique permettrait à Foucault, selon B. Han, « de penser de façon plus cohérente le statut de l'a priori historique lequel pourrait alors être défini comme une "époque " de l'être " (p. 99).

La deuxième section, la plus longue, est consacrée au tournant généalogique et aux nouveaux concepts qui l'accompagnent tels que ceux de "discipline", de « stratégie ", sans oublier évidemment celui de « pouvoir ». Désormais, il n'est plus question d'envisager le savoir dans une relative autonomie et en fonction seulement d'un a priori historique mais, selon une conception de l'interprétation empruntée à Nietzsche, d'interroger le type de volonté de vérité qui l'anime; ce qui conduit Fou- 
cault à examiner le rapport du savoir et du pouvoir, le nexus savoir-pouvoir. B. Han expose alors très clairement la nouveauté de l'articulation foucaldienne du pouvoir et du savoir par rapport aussi bien à Platon qu'à Bacon ou à Marx, à travers l'analyse des notions complémentaires d'objectivation et d'assujettissement. De ce point de vue, conformément à l'idée que toute technique d'assujettissement donne lieu à des procédures d'objectivation, Surveiller et punir rattache la naissance de l'homo criminalis et de la criminologie à des techniques telles que l'examen ou la surveillance.

Une telle imbrication du savoir et du pouvoir conduit à s'interroger sur la fonction des sciences humaines qui ne sauraient dans cette perspective libérer qui que ce soit; elle introduit, également, une conception nouvelle de la vérité qui ne recherche pas seulement les règles de l'acceptabilité - en vertu desquelles une proposition peut être jugée fausse - mais s'interroge également sur ce que Foucault dénomme le régime de la vérité. L'auteur souligne alors, de manière tout à fait pertinente, nous semble-t-il, et sans pour autant gommer les différences, la proximité de Foucault et de William James qui subordonnent, chacun à leur manière, la vérité à l'efficacité. En même temps, il faut bien reconnaître que cette notion de vérité ne va pas sans susciter de graves difficultés. Dans quelle mesure faut-il penser la vérité à partir de son rapport au pouvoir? Cette thèse concerne-t-elle toutes les vérités, y compris les vérités mathématiques? Dans l'expression « régime de vérité »s'agit-il d'un génitif subjectif ou objectif? Bref, force est d'admettre avec $B$. Han « qu'il devient très difficile dans ces conditions de savoir ce qu'est la vérité pour Foucault " (p. 233).

Au cours d'un entretien paru en avril 1984 dans Le Monde aujourd'hui, Foucault déclarait avoir toujours tourné autour du même problème, "c'est-à-dire les rapports entre le sujet, la vérité et la constitution de l'expérience ". Comme le montre B. Han, cette auto-interprétation rétrospective et la continuité qu'elle suggère sont largement artificielles. Car cette problématique appartient avant tout à la troisième période, et correspond au deuxième et au troisième volume de La Volonté de savoir. En effet, L'Archéologie du savoir rejetait fermement cette idée d'expérience à propos de la folie : une expérience de la folie supposerait un vaste sujet de l'histoire, avatar du sujet transcendantal; de même la notion de sujet a été dénoncée aussi bien par l'entreprise archéologique que par le point de vue généalogique. Tout cela est relativement bien connu. Reste en revanche à comprendre, selon une logique immanente à l'œuvre, les motifs de cette nouvelle étape centrée, plus précisément, sur la notion de subjectivation - qui serait à la fois « le processus réflexif par lequel le sujet forme une interprétation de ce qu'il est et se reconnaît en elle, et, d'autre part, l'action par laquelle il cherche à se transformer conformément à cette interprétation " (p. 271).

Mais s'agit-il bien, à travers cette dernière notion, d'un retour du sujet? Au cours de cette troisième section, B. Han pointe dans l'analyse foucaldienne de la subjectivation "une ambivalence fondamentale " liée au caractère constituant ou constitué du sujet. Ainsi les derniers textes de Foucault esquisseraient-ils un retour à l'humanisme sartrien à travers l'idée d'une libre création de soi. Mais, ce faisant, Foucault prendrait le risque "d'une régression en deçà des acquis généalogiques " (p. 276). Dès lors serait abandonnée l'interprétation de la subjectivation en référence aux pratiques de pouvoir et aux jeux de vérité anonymes. Par suite, un tel choix oblitérerait la possibilité d'asseoir l'archéogénéalogie foucaldienne sur l'ontologie heideggérienne; en d'autres termes, il interdirait définitivement tout rapprochement, 
comme le proposait déjà Hubert Dreyfus, entre l'Être heideggérien et le « pouvoir » foucaldien, entre les étapes de l'histoire de l'Être et celles de l'histoire foucaldienne de la vérité.

Quelques historiens de la philosophie reprocheront peut-être à cet ouvrage certaines simplifications ou " approximations". Il nous semble difficile de soutenir, par exemple, que pour la phénoménologie en général le sujet est dépourvu d'essence (p. 302) - y compris pour ce qui regarde la phénoménologie sartrienne : si l'existence précède l'essence, s'il n'y a pas de nature humaine, $L$ 'Être et le néant n'en décrit pas moins l'essence même de la réalité humaine à commencer par le circuit de l'ipséité en tant que structure essentielle de la liberté. De même lui reprochera-t-on peut-être de privilégier la recherche des ambiguïtés, des insuffisances et de multiplier à loisir les contradictions. En un mot, la lecture de B. Han n'est pas toujours très généreuse; et on en oublierait presque que l'œuvre de Foucault mérite sans aucun doute les nombreuses heures qu'elle-même y a consacrées.

L'Ontologie manquée de Michel Foucault n'en reste pas moins à bien des égards un travail convaincant. Tout d'abord, grâce à son effort d'élucidation conceptuelle, il offre la possibilité d'une lecture plus rigoureuse de Foucault; et le résultat est d'autant plus précieux qu'on doit bien reconnaître avec son auteur le « fort contraste entre le côté fouillé des analyses empiriques de Foucault et la grande imprécision de l'armature théorique sur laquelle celles-ci sont censées reposer" (p. 66). En outre, les critiques adressées nous paraissent souvent d'une grande pertinence. Par exemple, que peut bien être cet individu, qu'on ne saurait appeler un homme, qui rend pensable le processus de la subjectivation mais que, manifestement, le nominalisme foucaldien ne peut penser (p. 271-272)? Enfin, il permet une véritable mise en question philosophique des thèses de Foucault en privilégiant le rapport complexe de cette œuvre avec la phénoménologie au sens large et, plus particulièrement, avec l'ontologie herméneutique. À juste titre, s'il est vrai que Heidegger a toujours été pour Foucault «le philosophe essentiel ».

Philippe CABESTAN

Gérard Genette, L'Euvre de l'art. T. II : La relation esthétique. Paris, Seuil 1997. $14 \times 20,3,297$ p., bibliogr., index (Poétique).

Gérard Genette a développé depuis des années une cuvre qui relève à la fois de la théorie de la littérature, de la théorie de l'art et de l'esthétique. Son dernier livre, $L a$ Relation esthétique, est le deuxième volume d'un ouvrage dont l'ensemble est intitulé L'CEuvre de l'art. La première partie, Immanence et transcendance, examinait les questions ontologiques soulevées par la théorie de l'art. La seconde partie envisage l'esthétique comme une " anthropologie générale " (p. 11).

Ce projet est influencé par ce qu'on peut appeler l'esthétique analytique - le sous-ensemble « esthétique » de la philosophie analytique. Dans ses méthodes et ses attentes, l'esthétique analytique est aux antipodes de la tradition idéaliste allemande, 
si bien acclimatée sous les cieux français sous forme de lectures de Hegel, de Husserl, d'Adorno, de Heidegger. La tradition analytique favorise des analyses restreintes de thèses déterminées et leur discussion critique; elle insiste sur l'argumentation rigoureuse et exige (autant que possible) la clarté du discours philosophique. La tradition idéaliste vise l'interprétation globale philosophique de phénomènes artistiques plutôt qu'un décorticage analytique des thèses. La préférence de Genette pour le courant analytique, et ses représentants en esthétique (Frank Sibley, Monroe Beardsley, Nelson Goodman, Kendall Walton, par exemple), est justifiée, selon lui, par la différence entre l'intelligibilité des thèses qu'ils soutiennent, comparée aux proclamations invérifiables des tenants de la tradition spéculative allemande héritière des Romantiques et de Hegel.

On pourrait objecter que l'intelligibilité est relative, et non pas intrinsèque. Ce n'est vraisemblablement pas Genette qui dirait le contraire. Mais il est clair que pour qui a des doutes sur la pertinence de prétentions à saisir le Sens à l'œuvre dans l'Histoire de l'Esprit, quand il se manifeste dans l'Art ou ailleurs, pour qui l'idée d'un contenu spirituel des ouvres d'art saisi par une herméneutique philosophique est simplement un projet peu crédible, l'esthétique analytique sera un net changement de registre. Genette entend parler philosophiquement de l'art et du jugement esthétique sans les affirmations obligées sur la Vie, l'Histoire, le Sens, etc., d'un discours englobant, pathétique et grandiloquent!

Pour autant, l'intention de Genette n'est nullement de présenter les travaux des philosophes analytiques à un public français qui, en général, les ignore. Il propose sa propre thèse; il ne discute celles des autres que s'il lui semble expédient d'exposer la sienne sous forme critique. Sa thèse principale est celle du subjectivisme et du relativisme en esthétique. Son relativisme rejette tout pathos de l'absence de fondement objectif de nos jugements. Ce n'est pas quelque chose de négatif ou de métaphysiquement inquiétant. Genette insiste surtout sur l'idée que «l'appréciation esthétique est constitutivement objectiviste parce qu'elle ne peut renoncer à un corollaire d'objectivation sans se ruiner elle-même " (p. 106). Autrement dit, une appréciation esthétique se présente toujours comme objective. Mais c'est aussi toujours une illusion de la penser telle. Finalement, tout le livre consiste à tirer les conséquences du caractère à la fois illusoirement et constitutivement objectiviste de l'appréciation esthétique. Ainsi, après avoir présenté une conception de «l'attention esthétique " (une discussion de Goodman), de «l'appréciation esthétique » (dans laquelle s'affirme son subjectivisme relativiste), il analyse longuement l'idée de "fonction esthétique ».

Ce livre de Genette, comme le précédent, constitue pour l'esthétique, et singulièrement l'esthétique de langue française, un apport important. La façon délibérée dont Genette choisit de s'insérer dans la tradition analytique marque une nette modification du paysage esthétique français. On peut aussi penser que la culture de Genette, son choix d'exemples, son style d'écriture, sa liberté de ton sont des modèles pour tous ceux qui écrivent sur de tels sujets. Du reste, cela fait de la lecture de son livre un réel moment de plaisir intellectuel. Pour autant, on peut avoir des réserves sur la thèse générale qu'il soutient, celle du subjectivisme et du relativisme de l'appréciation esthétique. Voici trois raisons de douter de la possibilité de soutenir une telle thèse. 
D’une part, Genette développe sa thèse contre Hume. De façon générale, être humien revient à dire que les vérités que nous croyons nécessaires ne le sont pas à la façon dont on croit qu'elles le sont. Elles ne constituent que des conjonctions constantes, pas des relations intrinsèques ou des propriétés réelles des choses. La nécessité n'est que l'objectivation illusoire de nos habitudes. Même en esthétique, c'est cela que dit Hume. Soit la même chose que Genette, malgré ce que dit ce dernier. Mais, contrairement à Genette, Hume n'en tire pas une conséquence radicalement relativiste. Il peut, en effet, y avoir des experts qui savent mieux que d'autres, dont le jugement est plus sûr. Croire que si le jugement esthétique n'est pas le fait d'une nécessité réelle, s'il est illusoirement objectif, alors il est inévitablement relatif, c'est faire une implication incorrecte. Que les propriétés esthétiques ne soient pas réelles n'implique pas le relativisme que défend Genette.

D'autre part, la thèse de l'objectivisme illusoire de l'appréciation esthétique suppose que cette appréciation soit exprimée dans un jugement qui est systématiquement incorrect. On croit dire quelque chose au sujet d'un objet, on ne fait que présenter une préférence sans rapport réel avec cet objet. Le plus étonnant est que, selon Genette, même si je le sais, je continue à le faire. Le jugement esthétique serait alors une sorte de tromperie de soi-même : faire comme si on croyait quelque chose alors que manifestement on ne peut pas le croire. Genette le théoricien sait bien que l'objectivisme dont il fait preuve dans ses jugements esthétiques est une illusion, mais Genette l'esthète continue à faire preuve d'un objectivisme naïf. On a là une problématique analogue à celle de l'akrasia chez Aristote (c'est-à-dire agir contre son meilleur jugement). Et, dès lors, on a le même problème, dont le livre de Genette ne dit rien, alors qu'on aurait tant besoin d'explications.

À supposer tout de même que Genette ait raison : les croyances esthétiques sont irrationnelles dans la mesure où elles sont constitutivement illusoires. Or, nos croyances esthétiques ne sont sûrement pas isolées de nos autres croyances. Par exemple, mes croyances esthétiques au sujet d'un tableau, qu'il est triste et grandiose, ne sont vraisemblablement pas totalement indépendantes d'autres croyances au sujet de ce qu'il représente, de qui l'a peint, à quel moment, etc. Or, ces dernières croyances sont vraies ou fausses, mais pas constitutivement illusoires. Alors de deux choses l'une. Une solution consiste à isoler les croyances esthétiques qui n'ont rien à voir avec les autres croyances susceptibles d'être correctes ou pas. Dès lors, il y a un état mental esthétique spécifique. Cette affirmation est forte. On peut simplement se demander si l'argumentation de Genette est suffisante pour la soutenir. Kant le peut (même s'il ne soutient pas que cet état mental est irrationnel), car dans le cadre d'une analyse transcendantale de l'esprit, il fait de cet état mental spécifique, celui du plaisir esthétique, la condition de possibilité d'un type de jugement. Rien de cela chez Genette, mais pouvait-il s'en passer? Une simple anthropologie suffit-elle quand on a une thèse aussi forte ? Une seconde solution revient à soutenir qu'il n'y a pas d'état mental esthétique spécifique et complètement isolable de notre état mental général. Dès lors, seul un être foncièrement irrationnel peut avoir des croyances esthétiques, puisque avoir de telle croyances suppose que l'illusion soit le mode même de notre pensée. Genette serait-il prêt à soutenir une telle thèse?

Enfin, le problème général de ce livre, comme du précédent, c'est peut-être que Genette soutient des thèses qui semblent avoir certaines conséquences qu'il n'entend pas assumer complètement. Dans le précédent livre, l'affirmation selon 
laquelle il y a des entités idéales était ontologiquement moins anodine que Genette semblait le penser. Cette fois, les conséquences dans le domaine de la philosophie de l'esprit de sa conception de l'expérience esthétique ne sont pas si légères que cela...

Malgré ces réticences, la lecture du livre de Genette est hautement recommandable. Ce livre constitue, en effet, une magistrale défense d'une thèse au moins discutable, celle du relativisme esthétique. Trop souvent, les philosophes adoptent cette thèse comme allant de soi ou, au contraire, ils la rejettent de façon épidermique. On en a maintenant une formulation claire. Ce qui devrait réjouir tout le monde, ceux qui pensent que le relativisme esthétique est une thèse correcte, et ceux, comme l'auteur de cette recension, qui pensent qu'elle est incorrecte.

Roger Pourvet

Passion des formes. Dynamique qualitative, sémiophysique et intelligibilité. À René Thom. 2 vol. Sous la resp. de Michèle PorTe. Fontenay-aux-Roses/Paris, ENS Éditions Fontenay-St Cloud/Diff. Ophrys, 1994. $17 \times 24,5,821$ p., bibliogr. (Theoria).

Ce qui frappe d'emblée le lecteur de cet ouvrage considérable, c'est la manière toute particulière et audacieuse d'entendre la pluridisciplinarité : en premier lieu, elle réunit les savoirs que l'on croyait irréconciliables, selon le geste de René Thom lui-même dont le travail jette un pont entre le nombre et le discours, «les deux pentes naturelles du Logos ». Ainsi les mathématiques, la physique, la biologie et la philosophie, la linguistique, la psychanalyse, l'ethnologie sont représentées. En second lieu, elle ne renonce pas à la spécialisation des disciplines qu'elle réunit : il ne s'agit pas de présenter une somme, de faire le point de ce que nous savons ou pouvons savoir grâce à un jeu de complémentarité des sciences, mais bien plutôt de montrer ce que la pensée de Thom permet d'aborder autrement, ce qu'elle ouvre comme voies de recherche dans chaque spécialité. Bref, il est plus question dans ce livre de débats que de convergences, d'ouvertures que de bilans, de problèmes que de solutions.

Il ne faut pas s'étonner d'une telle « incertitude » puisqu'elle est la rançon d'une manière originale d'interroger la nature et l'homme à travers les événements physiques et psychiques : renouer le nombre au discours, au sens, c'est ouvrir un champ à la pensée, inconnu et médian, entre les sciences dites exactes ou «dures » et les autres, un entre-deux qui n'est pas une tentative de mariage forcé, un compromis, mais l'adoption d'un langage commun qui renonce autant à la formalisation mathématique qu'à la logique de la pensée ordinaire. Il n'entre point de naïveté dans une telle opération, car on sait bien que renoncer à la formalisation c'est du même coup perdre l'exactitude quantitative, le calcul comme mode opératoire et la rigueur méthodologique qui définissent la science classique. Et c'est le propre de la pensée thomienne que d'assumer une théorisation autre, qui prête le flanc aux critiques "scientifiques", et à laquelle on ne peut, sans être en contradiction avec les 
contemporains, donner le nom de « science ». De même, soustraire les événements psychiques ou sémantiques à la seule analyse de la pensée ordinaire pour les intégrer à une analyse morphologique géométriquement contrôlée, c'est dénier au discours la capacité à se comprendre lui-même, à se rendre intelligible par lui-même, remettre en question l'axiome de l'autonomie de l'esprit sur lequel se sont construites les sciences de l'homme. On comprendra dès lors les résistances, les doutes, voire les oppositions que rencontre un tel projet : toute science, sociologiquement et méthodologiquement bien établie, ne peut l'accueillir qu'au prix d'une transformation radicale, du point de vue des visées et des méthodes. C'est d'un nouveau paradigme qu'il s'agit, qui met en jeu ce que connaître veut dire.

En effet, pourquoi se délier de la formalisation sinon parce qu'elle tend à l'insignifiance? Dire comme Thom que « l'insignifiance est le bord de la science », c'est mettre en discussion l'individuation de la science, qui pour un topologue, est affaire de frontières : paradoxalement, plus une science est rigoureuse, opératoire et exacte, moins elle est une science. On dira que c'est une question de mots, qu'on confond ici la connaissance et la science constituée. Or, il n'est justement pas question ici de connaissance, s'il est vrai qu'une science constituée, rigoureuse, ne cesse d'étendre le champ de ses connaissances, mais d'intelligibilité ou de sens : s'il y a un jeu de mot, il porte sur l'insignifiance, qui veut dire tout ensemble, le corrélat de l'opération de formalisation (que l'on pense à la logique formelle qui s'est construite sur la négligence du sens des propositions pour s'attacher aux relations) et ce qui ne provoque pas l'intérêt. Ainsi, le véritable critère d'une science serait sa capacité à dire ce qu'elle connaît, et à emporter l'adhésion de tout un chacun sur l'importance et l'intérêt pour la pensée de ce qu'elle découvre. En revanche, de l'autre côté, recourir à une modélisation géométrique des activités psychiques au détriment de la logique ordinaire se justifie seulement par une raison inverse : il n'y a d'intérêt à formaliser la pensée verbale que si on se libère d'une logique du sens commun et que l'on parvient par là-même à formuler des assertions qu'elle seule ne permet pas. À ce titre, Thom a l'habitude de citer «le prédateur affamé est sa proie " comme assertion «translogique ", qu'il voit à la base de l'embryologie animale. Mais on pensera aussi à ce que la modélisation géométrique permet de découvrir comme analogies, inaccessibles à la seule imagination verbale et permettant de rapprocher des situations ou des événements qui n'offrent pas de ressemblances visibles ou de proximités structurelles.

Aussi doit-on penser que l'opposition du qualitatif et du quantitatif, qui bien entendu est l'objet d'un débat, n'a pas pour pierre de touche la question de la formalisation mais celle, au-delà de ce que techniquement il convient d'appeler qualitatif et quantitatif, de l'intelligibilité. En ce sens, la sémiophysique, élaborée dans le cadre des mathématiques qualitatives (dynamique qualitative et théories des catastrophes) vise à rendre intelligible les phénomènes, plutôt qu'à les prédire, pour autant qu'elle suppose une théorie du sens.

La sémiophysique est ce nouveau langage qui se propose de formaliser géométriquement le sens grâce à la dynamique qualitative et à la théorie des catastrophes. Il s'agit d'une physique du sens, où il faut entendre la phusis grecque, telle qu'Aristote a pu la concevoir à partir des concepts de forme, de substrat et de génération. Ce recours au Stagirite est peut-être l'un des traits philosophiques les plus marquants du travail élaboré ici : d'une part, parce que cette nouvelle philosophie natu- 
relle est un essai d'ontologie, dont on regrettera peut-être qu'il ait donné lieu à une interprétation historienne plutôt que philosophique dans le présent livre. D'autre part, parce que la pensée d'Aristote prend au sérieux le problème de la généricité, qui, dans les travaux de Pierre Aubenque, Bernard Besnier et Pierre Pellegrin, lie l'analyse morphogénétique (génération, croissance, stabilité et corruption d'une forme) à l'affirmation du primat ontologique du continu sur le discret, que revendique la sémiophysique. S'il est vrai que le genre, selon Aristote, se distingue de la forme en ce qu'indifférencié, il est un engendrement continu et spécifié d'éléments différenciés, alors la généricité est ce qui permet de penser le primat ontologique du continu.

Dès lors, il semble que l'enjeu soit le suivant : au-delà de la théorie des catastrophes dont l'aspect statique, local et irréversible nécessite un usage prudent et somme toute difficile, il s'agit de se donner les moyens de considérer des dynamiques d'engendrement plus générales que celles des catastrophes élémentaires. C'est pourquoi, la sémiophysique établit un typologie des événements physiques comme sémantiques, à partir des concepts généraux de saillances et de prégnances. Ces concepts visent à décrire les phénomènes, qui sont par et selon des formes (analyse qualitative) et qui évoluent dans et selon le temps (analyse dynamique). Ils rendent compte de leur individuation physique qui est le domaine connexe d'espacetemps qu'un être occupe ou de leur individuation sémantique, le doublet extension/ intension du concept (la forme-saillance) mais aussi de leurs relations, l'investissement d'une forme par une autre, les phénomènes de forme-source ou bien de formeflux (la forme-prégnance). Cependant, on voit d'emblée que cela ne va pas sans problèmes : d'une part, le physicien peut rétorquer qu'il ne travaille jamais sur des formes, que les phénomènes qu'il étudie ne relèvent pas de structures stables; d'autre part, on peut douter plus généralement que le sens gagne beaucoup à être analysé en termes de formes qui ne peuvent être qu'unidimensionnelles ou linéaires. Or, dans ce dernier cas, comprendre en quoi cette formalisation géométrique des phénomènes sémantiques présente un intérêt exige qu'on aborde l'analogie fondatrice de la sémiophysique : considérer tout phénomène sémantique (une proposition linguistique ou une déduction logique) du point de vue de la généricité, la déduction comme génération, le choix linguistique d'un mot par exemple du point de vue de la théorie des catastrophes. Les nombreuses contributions des linguistes (P. A. Brandt, H. Seiler, B. Victorri ou G. Vincent) ou celle de Jean Petitot pour les sciences cognitives permettent de découvrir la portée de telles analyses.

On comprendra désormais que la sémiophysique vise l'intelligibilité des phénomènes physiques ou psychiques par des modèles morphogénétiques, pour autant que le sens tient tout entier dans sa propre généricité et qu'il ne se découvre qu'à ceux qui en étudient la génération. Davantage, le pari d'expliquer les phénomènes par des modèles géométriques n'est pas seulement l'affirmation du continu mais révèle qu'il entre beaucoup de vision dans la compréhension selon Thom, ou encore que la métaphore est maîtresse du sens. À ce titre, les articles de G. Châtelet, B. Teissier ou Fernando Gil soumettent à la critique cette "ontologie de l'analogie », théorie du sens que met à l'œuvre la sémiophysique et ses rapports pour le moins conflictuels avec l'épistémologie moderne. Ainsi, le conflit qui oppose ce programme de recherche, dont Petitot dit qu'il a pour objet la "naturalisation du sens " et dont témoignent certaines contributions du livre Passion des formes, au paradigme de la 
science moderne, a non seulement pour ultime raison une mésentente sur ce que signifie «intelligibilité », mais encore révèle que la sémiophysique est tout ensemble la mise en ouvre d'un langage et l'élaboration philosophique des conditions de toute explication, de toute tentative de « rendre intelligible » les phénomènes. C'est pourquoi, en liant indissolublement une théorie du sens (philosophique) à une théorie de l'explication (épistémologique), elle se démarque des pratiques scientifiques constituées en les soumettant à une double et redoutable épreuve : savoir dire ce qu'on connaît et savoir expliquer ce qu'on dit.

On ne peut mettre un terme à cette note nécessairement lacunaire sans recommander la présentation exemplaire de Michèle Porte, tant elle élucide les relations implicites et les apports conjugués des différents travaux publiés dans ce livre. S'il fallait simplement énoncer les signes de reconnaissance de cette nouvelle philosophie naturelle, on pourrait dire d'elle d'une part, qu'elle abandonne l'objectif d'une grande précision quantitative pour celui d'une description géométriquement rigoureuse de la forme, et qu'elle rattache d'autre part l'évolution du système au devenir de la forme dans et par le temps et non aux changements quantitatifs. Son ambition serait de disposer d'une philosophie des types (contre l'approche génétique en biologie par exemple, cf. l'article de R. Chandebois) et d'une théorie du sens construite sur les formes-prégnances. On aura compris que l'entreprise ne manque pas de ressources ni d'intérêt puisqu'elle promet le mariage heureux de la science et de l'imagination.

Pierre-Louis Autin

Bas C. Van Fraassen, Lois et symétrie. Prés. et trad. par Catherine Chevalley. Paris, Vrin, 1994. 13,5 × 21,5, 520 p., bibliogr., index (Mathesis).

Si l'on considère cet ouvrage dans son ensemble, les intentions de l'auteur paraissent assez bien définies. Il s'agit de délivrer le lecteur de certaines idées traditionnelles dont les philosophes de la science auraient cru à tort qu'elles étaient nécessaires et suffisantes pour atteindre leur but principal : échapper au scepticisme tout en se gardant des illusions métaphysiques sur les fondements de la certitude.

L'un des concepts visés est celui de « loi de la nature »; son analyse et sa justification s'appuient traditionnellement sur les notions d'universalité, de nécessité, de possibilité dont l'examen attentif révèle qu'elles ne cessent de se compliquer et de se dérober à mesure qu'on s'efforce de les saisir par des définitions et des principes rigoureux.

Un autre des préjugés dénoncés par Bas C. Van Fraassen est l'hypothèse qu'une analyse rigoureuse du langage de la connaissance pourrait suffire à en établir la théorie.

Intervient, enfin, dans son enquête critique une discussion très serrée de la notion de « probabilité logique » dont il montre soigneusement qu'elle est finalement insaisissable, discussion qui, reprise dans diverses perspectives, est sans doute l'un des éléments les plus originaux du livre. 
Mais la dénonciation de ces certitudes illusoires laisse, selon l'auteur, des ressources disponibles, en fait mieux utilisées jusqu'ici dans la pratique des sciences que dans celle de la philosophie; ces ressources il les trouve dans l'esquisse d'une théorie sémantique de la connaissance, appuyée sur le concept de « modèle », explorant ainsi une voie intéressante dans l'analyse du travail heuristique et démonstratif de la science (en évitant justement les inconvénients de la dichotomie entre découverte et démonstration). Et, à cet égard, la notion de symétrie, considérée aussi bien comme instrument de découverte que comme guide de la démonstration, joue pour l'auteur un rôle important qu'il s'efforce, à l'aide de nombreux exemples, de mettre en évidence.

Cependant, le lecteur éventuel aurait tort de trop se fier à une présentation aussi élémentaire du programme de Van Fraassen. Car si l'ouvrage abonde en aperçus intéressants, en dicussions subtiles et rigoureuses de questions importantes, son cheminement est souvent difficile à suivre et sa démarche déconcertante.

La critique de la notion de « loi de la nature » est assurément pertinente mais elle est loin d'être convaincante; à notre avis, les arguments de l'auteur ne suffisent nullement à prouver que la recherche des lois de la nature ne reste pas, en fait et en droit (compte tenu de l'évolution du vocabulaire qui fait que les savants contemporains préferent parler, dans certains contextes de l'« équation de Poisson » plutôt que de la « loi newtonienne de la gravitation »), l'un des buts les plus importants de la recherche contemporaine; et on se demande quelle place des lois reconnues comme telles par les physiciens, par exemple les énoncés fondamentaux de l'énergétique, peuvent prendre dans le système sans lois de Van Fraassen, alors qu'ils sont justement indépendants de tout modèle, et que l'un au moins ne doit rien au concept de symétrie.

La critique de la notion de nécessité ne semble pas en outre décisive, notamment parce que le caractère conditionnel qu'il a dans son application aux lois de la nature, telle au moins que la physique théorique l'envisage, n'est finalement pas mis clairement en évidence dans les discussions de l'auteur qui n'a pas cherché explicitement à élucider les rapports entre la nécessité logique des systèmes formels, la nécessité inconditionnelle qu'affirment les systèmes de la nature d'inspiration théologique ou métaphysique, et la nécessité naturelle que suppose, toujours partiellement et relativement, mais inévitablement, la recherche des lois de la nature.

L'auteur concède beaucoup de place à la mode épistémologique actuelle des " mondes possibles ", moyen d'exprimer la distance irréductible entre les visées du discours théorique et la réalité naturelle qui n'est qu'une partie de son champ sémantique; discussion parfois intéressante mais finalement décevante; le maniement de ces entités fictives éclaire-t-elle d'un jour nouveau les notions intuitives de possibilité et de contingence? À lire l'ouvrage, on n'en est pas convaincu.

Catherine Chevalley a réussi à donner de ce difficile ouvrage une traduction française à la fois lisible (ce qui ne va nullement de soi pour un texte de ce genre...) et solidement justifiée par une note ad hoc. Elle offre en outre au lecteur une soigneuse présentation qui analyse l'ouvrage en le situant très précisément dans le cadre de la philosophie contemporaine de tradition analytique. 
Vernon Pratt, Machines à penser. Une histoire de l'intelligence artificielle. Trad. de l'américain par Christian Puech. Paris, Presses universitaires de France, 1995. $15 \times 21,7$, vill-299 p., index (Sciences Modernités Philosophies).

Vernon Pratt retrace l'évolution des idées et des techniques qui ont permis, au milieu de notre siècle, l'émergence de l'idée d'intelligence artificielle (IA). Selon lui, trois conditions devaient être réunies pour que puisse être formulé le projet contemporain de construction d'une machine pensante. La première est la distance entre le sujet connaissant et l'objet connu établie au xvII siècle grâce à une nouvelle conception de la pensée comme manipulation de représentations; la deuxième est le développement, à la fin du xIX ${ }^{\mathrm{e}}$ siècle, d'une logique symbolique comme l'instrument de cette manipulation; la troisième, enfin, est la construction, au $\mathrm{xx}^{\mathrm{e}}$ siècle, d'automates électroniques, incarnations d'une machine universelle capable de mettre en œuvre toute manipulation de symboles analysable en une suite d'étapes finies. Cette thèse somme toute banale est soigneusement documentée et présentée de façon originale.

L'analyse de Pratt est centrée sur trois projets paradigmatiques attribués respectivement à Leibniz, Charles Babbage et Alan Turing ${ }^{1}$. Cependant, ce ne sont pas ces trois projets en eux-mêmes qui intéressent l'auteur, autant que le statut de balise qu'il leur confère dans un paysage conceptuel dans lequel les idées s'organisent plus morphologiquement que chronologiquement. Ainsi, l'ouvrage met en jeu trois acteurs qui, paradoxalement, ne sont pas Leibniz, Babbage et Turing, mais trois concepts que les projets associés à ces trois personnages mettent en interaction chacun à sa manière, dans le contexte du paysage intellectuel de son époque : le concept de représentation, le concept de formalisation et le concept d'automatisation.

Là où les Anciens voyaient la participation de la pensée à la forme de l'objet, les Modernes voient la présence dans l'esprit d'une idée qui tient lieu d'une réalité non mentale. La connaissance a changé de nature : à la communion des essences se substitue la manipulation des représentations. Pratt voit dans cette révolution épistémologique la condition nécessaire, sinon suffisante, de la formulation d'un projet de construction d'une machine pensante. Cette thèse permet de donner toute leur pertinence aux descriptions détaillées que l'auteur nous fournit de l'organisation interne des premiers automates calculateurs. En effet, bien que l'auteur ne le dise pas explicitement, il ressort clairement de ces descriptions et des croquis qui les accompagnent que les premières machines à calculer, dans leur structure, c'est-àdire dans l'agencement de leurs parties, sont des représentations du système décimal, de sorte que des manipulations de cette structure, c'est-à-dire des opérations mécaniques sur cette structure, constituent en elles-mêmes des manipulations de (des opérations sur) ce que cette structure représente. La puissance des ordinateurs modernes par rapport à ces premiers calculateurs tient d'ailleurs en partie au fait que

1. L'ouvrage est d'ailleurs divisé en trois parties intitulées "Leibniz' project », "Babbage's project » et « Turing's project ». Il est regrettable que le traducteur ait pris la liberté de traduire ces trois titres respectivement par «Le projet leibnizien ", "L'entreprise de Babbage » et «La perspective de Turing », occultant ainsi le fait qu'il s'agit bien aux yeux de l'auteur de trois projets avec tout ce que la notion de projet véhicule d'élan créatif, voire même prophétique. En ce qui concerne la faiblesse de la traduction, voir infra n. 4. 
leur architecture n'est plus directement représentative d'un domaine spécifique. La fonction représentative est assumée par la base de connaissances et les opérations définies programmatiquement sur elle. C'est en cela que ces nouveaux automates sont des machines universelles au sens de Turing.

De nos jours, l'approche dominante en intelligence artificielle est encore redevable - certains diraient tributaire - de la conception moderne de la pensée comme manipulation de représentations, comme en témoigne l'hypothèse connue sous le nom d' " Hypothèse du système physique de symboles » ou encore « Hypothèse représentationnelle », formulée par les deux pionniers de l'IA, Allen Newell et Herbert Simon : «L'étude de la logique et des ordinateurs nous a révélé que l'intelligence réside dans des systèmes physiques de symboles. [...] Un système de symboles est une collection de structures (patterns) et de processus, ceux-ci pouvant produire, détruire et modifier celles-là. [...] La propriété la plus importante de ces structures est qu'elles peuvent désigner des objets, des processus et d'autres structures et que, lorsqu'elles désignent des processus, elles peuvent être interprétées ${ }^{2}$. » Cette hypothèse postule que l'intelligence est dans la structure de l'arrangement des symboles et les opérations sur cette structure, indépendamment du médium utilisé pour les matérialiser, d'où la possibilité, en principe, de construire un système intelligent.

Pratt parle longuement des premiers travaux de Newell et Simon, en particulier du Logic Theorist, un système de preuve automatique de théorèmes logiques, et du GPS, un système général de résolution de problème (General Problem Solver), mais il ne fait jamais référence à l'hypothèse représentationnelle et ne présente que les aspects algorithmiques des travaux contemporains en IA. Il est vrai que dans la troisième partie de l'ouvrage consacrée au projet de Turing, l'auteur limite volontairement, mais à tort, son analyse aux premières années de l'IA pendant lesquelles l'importance des connaissances et de leur représentation dans les systèmes intelligents (systèmes représentationnels au second degré, puisqu'ils représentent des représentations) n'a pas été d'emblée reconnue comme le souligne Edward Feigenbaum : « Le premier principe de l'ingénierie des connaissances est que le pouvoir de résolution de problème démontré par la performance d'un agent intelligent est principalement la conséquence de sa base de connaissances et seulement secondairement de la méthode d'inférence employée. [...] C'est là un résultat important qui n'a pourtant été compris que récemment en IA. Pendant longtemps l'IA s'est focalisée presque exclusivement sur le développement de méthodes d'inférence ingénieuses; presque n'importe quelle méthode fera l'affaire. La puissance réside dans la connaissance ${ }^{3}$."

La problématique de la représentation des connaissances est donc essentielle en IA, du moins dans le courant symbolique (par opposition au courant connexionniste) qui est précisément celui auquel l'auteur fait référence. En effet, même si on sait que toutes les représentations qui ont la même puissance expressive sont en

2. Allen Newell et Herbert Simon, ACM Turing award lecture. « Computer science as empirical enquiry. Symbol and search », Communication of the $A C M$, vol. 19, 3, 1976, p. 113126.

3. Cité in Artificial Intelligence, structures and strategies for complex problem solving, G. F. Luger et W. A. Stubblefield, Benjamin/Cummings, 1993, p. 308. 
théorie équivalentes, la structure de la représentation joue un rôle déterminant dans les possibilités de manipulation qu'elle offre en pratique. Pratt donne implicitement un exemple de ce phénomène quand il rappelle l'importance qu'a eue, pour l'arithmétique, l'adoption de la notation arabe à la place de la notation romaine. Les deux notations sont des représentations adéquates des chiffres en base 10 et en cela leur puissance expressive est la même. Cependant, la notation romaine ne représente explicitement ni le 0 ni la relation entre les multiples de la base. En conséquence, dans cette représentation, les additions et les soustractions se font difficilement et les multiplications représentent un défi quasi insurmontable. La notation arabe au contraire, outre le fait de représenter le zéro, permet (comme d'ailleurs le boulier chinois), d'associer la position que le symbole occupe dans la combinaison des symboles avec un multiple de la base et libère de ce fait les manipulations arithmétiques.

Il est dommage que la problématique de la représentation des connaissances, pourtant reconnue comme déterminante par l'auteur dans son analyse du projet de Leibniz, soit presque totalement ignorée dans l'analyse des deux autres projets. De plus, justement parce que, dans les systèmes informatiques modernes, le fardeau de la représentation des connaissances s'est déplacé du matériel au logiciel, la description détaillée de l'architecture des automates, si intéressante dans le cas des premières machines à calculer, est non pertinente dans le cas des ordinateurs modernes. Insensibles à ce changement de perspective, l'auteur consacre pourtant de longues pages fastidieuses à la description de l'architecture interne des premiers ordinateurs.

L'automatisation de la pensée ne repose pas sur la construction d'un modèle physique de son objet (ce qu'étaient les premières machines à calculer pour le calcul arithmétique), mais sur la possibilité de construire un modèle logique de la pensée elle-même, ce dont Pratt a eu l'intuition lorsqu'il qualifie le projet de Turing de «projet d'une théorie formelle de l'esprit » (p. 287) - intuition cependant immédiatement banalisée quand il ajoute : «Les pensées se dissolvent dans un ensemble de sous-programmes imbriqués. " L'idée de modéliser la nature est une idée moderne qui va de pair avec l'idée de représentation; l'idée de modéliser la pensée est une idée contemporaine qui va de pair avec l'idée de formalisation. Le formalisme repose sur la possibilité de produire des systèmes de symboles interprétables mais non nécessairement interprétés, et, qui plus et, sur la possibilité d'une multiplicité d'interprétations concurrentes. C'est ici qu'intervient la logique, et plus particulièrement la logique symbolique telle qu'elle s'est développée à la fin du Xix siècle. Ce que le formalisme apporte, par la dissociation qui le caractérise entre la forme et le contenu, c'est une nouvelle façon de voir le concept. Avant Kant, les concepts étaient vus essentiellement comme des listes de propriétés en vertu desquelles la réalité pouvait être répertoriée et classifiée; après Kant, et plus encore après Frege, les concepts sont vus comme des formes qui rendent possible l'intelligibilité. D'où l'ouverture de tout un nouveau champ d'investigation encore à l'heure actuelle à peine exploré : quelle est la structure de nos concepts? (Et non « quelles sont les propriétés de ce qu'ils représentent? ».) Il est à se demander cependant si la logique formelle symbolique au sens que Charles S. Peirce donne à ce terme est un instrument adéquat pour une telle investigation. Le pessimisme de l'auteur quant aux chances de succès du projet de Turing tient peut-être au fait qu'il ignore complètement la possibilité de développer le formalisme dans une autre direction, celle d'une logique formelle iconique dont les signes dans leur structure interne 
refléteraient la structure de nos concepts, signes que la machine serait compétente à manipuler de façon significative sans que l'on ait besoin de postuler en elle la capacité intentionnelle de les interpréter. L'exploration d'une telle hypothèse déborde cependant largement le cadre de ce compte rendu.

Ce livre, malgré certaines faiblesses, a la plus grande vertu qu'un livre puisse avoir : celle de susciter la réflexion. Ce compte rendu ne prétend pas être toujours fidèle aux idées de l'auteur. Puisant dans la richesse du matériel rassemblé par Vernon Pratt, il est autant une réflexion sur le livre qu'il aurait pu écrire ou qu'il a écrit en filigrane, que sur le livre qu'il nous a de fait livré.

Notons pour terminer que la traduction française (de l'anglais et non de l'américain, contrairement à l'indication de la page de garde) laisse beaucoup à désirer. Le style de Vernon Pratt est simple, direct et sans prétention; la traduction au contraire est empesée, parfois pédante et qui plus est, pas toujours exacte ni fidèle ${ }^{4}$. La première de ces infidélités, et non la moindre, est d'avoir traduit le sous-titre « An Evolution of Artificial Intelligence " par "Une histoire de l'intelligence artificielle " alors que l'auteur tient à souligner que l'intelligence artificielle n'a pas à proprement parler d'histoire, elle n'a que des projets dont les objectifs et les stratégies peuvent être rapprochés. Il est dommage aussi que l'éditeur français ait choisi de ne pas reproduire les nombreuses illustrations et photos qui accompagnent le texte dans sa version originale. Loin d'être accessoires, elles font partie intégrante d'une démarche que l'auteur lui-même qualifie d'archéologique au sens de Foucault.

Maryvonne LONGEART

4. Dans certains cas, la traduction ne manifeste qu'une déficience technique du traducteur et ne porte pas à conséquence du point de vue du fond. C'est le cas, par ex., quand «doubleentry book-keeping " est traduit par «comptabilité à double entrée " au lieu de « comptabilité en partie double » qui est l'appellation exacte de cette technique; ou encore quand " the intelligence of the programmer " est traduit par «l'intelligence du programmateur " au lieu de «l'intelligence du programmeur " (on parle du programmateur d'un appareil électroménager ou du programmateur d'une activité culturelle, mais du programmeur d'un système informatique !). Mais, dans d'autres cas, la négligence est plus grave et frise le contresens. C'est le cas, par ex., de la traduction de l'expression « the idea that life can be changed par « l'idée que la vie peut changer » au lieu de « l'idée que la vie peut être changée »; de même, au début du chap. $x v$, on lit « le théorème " $\mathrm{Si}$ ( $p$ et q vrais simultanément)" " au lieu de « le théorème " $\mathrm{Si}$ ( $\mathrm{p}$ et $\mathrm{q}$ vrais simultanément) alors $p$ " ". Ce ne sont là que quelques exemples, on pourrait en citer bien d'autres. Pour rendre justice aux idées de l'auteur, il est souvent nécessaire de consulter le texte original et mon conseil, à quiconque serait vraiment intéressé par l'ouvrage de Vernon Pratt, serait, si possible, de le lire dans sa version originale. 
Frederic ScHICK, Making choices. A recasting of decision theory. Cambridge, Cambridge University Press, 1997. $14 \times 21,5$, v1-163 p., index.

Ce petit ouvrage fort bien conçu a les apparences d'un manuel d'introduction élémentaire à la théorie de la décision, et il peut assurément servir à cet usage. Il est cependant dépourvu de l'appareil mathématique qui caractérise d'autres livres de synthèse ou d'introduction (par exemple, pour citer les meilleurs, Games and decisions de R. D. Luce et H. Raiffa, ou Decision Theory de S. French). Ce parti pris "littéraire » ne saurait étonner, car l'auteur a surtout voulu donner une présentation du sens et de la portée de la théorie moderne de la décision, envisagée dans la perspective de la philosophie de l'action et du langage de l'action. Telle est précisément l'ambition qui fait défaut à tant de manuels de facture conventionnelle. Le pari est tenu, grâce à un choix judicieux d'exemples finement analysés, empruntés à la littérature, à l'histoire ou à l'art cinématographique (bien loin des fastidieux exemples imaginaires et calculatoires qui font d'ordinaire les délices de la théorie de la décision).

Si le style est original, le parcours est classique : le lecteur est conduit de l'analyse des choix individuels à celle des choix collectifs, en passant par la théorie des jeux et l'étude des conflits. S'inscrivant sans détour dans une perspective individualiste, l'auteur ne s'étend guère sur les homologies et les prolongements qui permettent de présenter la théorie des choix collectifs comme une extension, vers le social et le politique, du domaine d'application des concepts et des critères de rationalité qui ont permis d'élaborer, sur trois siècles, la théorie du choix individuel rationnel. Rien dans la problématique retenue n'invitait à emprunter cette voie. On doit toutefois faire observer que la démarche de l'auteur le dissuade d'approfondir la réflexion sur un problème essentiel : le statut des principes de rationalité dans la formation d'un jugement sur l'existence collective. De même, le lecteur n'aperçoit pas très clairement les raisons qui ont conduit à la constitution, au $\mathrm{xx}^{\mathrm{e}}$ siècle en particulier, d'une science formelle et systématique de l'action et des normes. Le talent de Frederic Schick dans l'analyse des exemples concrets fait oublier le labeur souvent obscur des théoriciens.

Le public francophone, grâce aux travaux de Maurice Allais, Bertrand SaintSernin, Jean-Pierre Séris et Gilles-Gaston Granger (pour ne citer que quelques noms), n'aura aucun mal à se familiariser avec les analyses que propose Schick des relations entre choix et risque, utilité et probabilité, jeux stratégiques et coopération. ll sera vraisemblablement plus troublé de ne trouver ici aucun écho des débats qui ont eu lieu (en France, notamment) au cours des dernières années, et qui ont déterminé l'évolution de la méthodologie dite «individualiste" dans les sciences sociales et dans la théorie morale et politique, selon trois axes principaux : la prise en compte dans la perspective d'un individualisme méthodologique élargi, des aspects cognitifs et axiologiques de l'action (chez Raymond Boudon, en particulier); une certaine reprise du dialogue, voire le début d'un rapprochement, entre l'individualisme méthodologique et d'autres tendances de l'analyse sociale, dans la mesure où ces dernières accordent elles aussi un rôle important à l'action, comme le montrent l'Histoire de la théorie sociologique de Pierre Demeulenaere (Paris, Hachette Éducation, 1997), plusieurs textes récents de Pierre Bourdieu, et la paru- 
tion du volume collectif Structure, système, champ et théorie du sujet (dir. Tory Andreani et Merahem Rosen, Paris, L'Harmattan, 1997); enfin, une interrogation nouvelle sur les rapports entre l'explication de l'action en termes de choix rationnel et de «bonnes raisons » d'une part, et la problématique des «causes de l'action » d'autre part, sur la toile de fond d'un " naturalisme » instruit des neurosciences.

Il faut cependant avouer que l'auteur se rapproche, à un degré considérable, des problématiques «continentales » récentes de la «rationalité cognitive » (ou, dans les termes d'une autre tradition sociologique, de l'idée d'un versant cognitif de la genèse de l' " habitus ») et de la « rationalité axiologique ", à travers ce qui constitue l'un des traits distinctifs du livre : le statut éminent accordé, dans l'analyse de la décision individuelle, aux "manières de voir " (seeings) ou «manières de comprendre » (understandings). Dans les deux cas, il s'agit bien de chercher à préciser l'incidence sur les choix et la conduite de la manière (naïve ou savante) de penser l'univers naturel ou social, et tout d'abord l'action elle-même, comme le montre l'exemple (repris de R. J. Lifton) du tragique changement d'attitudes des médecins des camps nazis (du refus initial à l'acceptation, après un surcroît d'endoctrinement, de leur atroce besogne). Cette tentative reflète bien la direction générale réellement nouvelle que prend la théorie de la décision en cette fin de siècle : l'effort d'intégration des aspects prudentiels, épistémiques et axiologiques en un seul corps de doctrine est désormais la règle, ce qui n'oblige pas à renoncer au projet d'élucidation d'une rationalité propre à la pratique. L' « agent » de la science des choix n'est plus le calculateur borné que l'on a trop décrié : il comprend désormais les raisonnements des épistémologues et des moralistes, puisqu'il se montre aussi rationnel dans la sélection des théories et des normes guidant la conduite que dans la recherche instrumentale des moyens efficaces conduisant à la réalisation de ses objectifs.

Les premiers exemples de l'ouvrage (un cas de conscience dans le contexte du maccartisme, la décision de subir une cure psychanalytique, le « choix de Sophie ") suscitent l'interrogation sur la nature même du choix : en quel sens peut-on évoquer un changement d'état du sujet entre le temps de la délibération, celui de la résolution, celui de l'action enfin? Le rapport entre le sujet et ses valeurs est-il constitutif, de sorte que le mépris de ses propres valeurs mettrait en cause l'unité de la personne, ou bien s'agit-il d'un rapport extérieur? Pourquoi peut-on, par la magie des mots, se laisser convaincre de se lancer dans une aventure personnelle susceptible de conduire à une révision de ses propres valeurs? Qu'est-ce qui distingue l'action réellement choisie de celle qui ne l'est pas? Dans la perspective de Schick, ces problèmes mettent en jeu une forme d'ouverture d'esprit : derrière l'énumération des « options » si familière au théoricien de la décision se cache une faculté - celle de se représenter plusieurs options comme des possibilités réelles.

De plus, l'auteur montre de manière convaincante que l'on ne parvient à prendre au sérieux l'importance de cette représentation des choix que si l'on renonce tout d'abord aux analyses purement hédonistes, selon lesquelles l'agent ne peut choisir que ce qui lui plaît le mieux (conception qui sous-tend, d'une manière purement conventionnelle il est vrai, la théorie moderne des indices d'utilité). Il faut, au contraire, que la théorie apprenne à donner tout son sens au fait que l'agent peut parfaitement choisir, pour des raisons autres que l'agrément personnel, ce qui ne lui plaît pas. Le vouloir lui-même résulte d'un choix, et le théoricien de la décision doit 
s'intéresser à ce qui dispose à vouloir, sans se contenter d'une identification de principe entre ce qui est voulu et ce qui est préféré.

Se situant de manière critique par rapport à la doctrine aujourd'hui dominante, d'ascendance humienne, selon laquelle l'explication de l'action procède toujours de la connaissance et de la combinaison des croyances et des désirs de l'agent (dont le statut de "fondements de l'action " est admis), l'auteur tente de montrer que l'existence de «bonnes raisons » de l'action ne suffit jamais à garantir que l'action soit effectivement voulue, autrement dit, que la volonté soit orientée (sans " faiblesse de la volonté ») dans le sens de l'action considérée. C'est aussi une manière de se situer face à Davidson : les raisons de l'action n'en sont pas les causes, car « il manque quelque chose » aux raisons si la volonté n'intervient pas de manière active. De plus, l'importance accordée aux « manières de voir » conduit à une structure tripartite des états mentaux : les raisons concernent la volonté de faire des choses d'une certaine sorte, la croyance que certaines actions sont de cette sorte, et une manière de considérer ces actions d'une façon telle que l'on puisse avoir cette croyance. Cette approche originale et intéressante repose sur une hypothèse qui peut être contestée : les "manières de voir " ne se laissent pas réduire à des croyances.

Le ton de l'ouvrage est simple et familier, ce qui facilite la compréhension des problèmes philosophiques réels. Les développements proposés, notamment autour de notions telles que les "manières de voir ", les émotions, la résolution des dilemmes moraux, les regrets, la dévalorisation du futur et les excuses, favorisent le rapprochement avec la psychologie. Par la pertinence des exemples et la subtilité des analyses, ce livre constitue une introduction très vivante aux problèmes actuels de l'analyse de l'action et de la philosophie du choix rationnel.

Emmanuel PiCAVET

Alain Prochiantz, La Biologie dans le boudoir. Paris, Odile Jacob, 1995. 14 × 21,5, $157 \mathrm{p}$.

La Biologie dans le boudoir a pour enjeu de souligner le caractère fondamental des notions de forme et d'individu en biologie. Pour le neurobiologiste Alain Prochiantz, il s'agit de définir l'objet propre de la biologie et de délimiter strictement ses champs de compétence. En exhibant les liens qui unissent désormais génétique, embryologie, neurobiologie et théorie de l'évolution grâce à l'indentification des gènes de développement, l'auteur affirme, à l'aide de la notion d'individuation, la spécificité du vivant et défend l'autonomie de la biologie face à la physique et à une compréhension exclusivement mathématisée du vivant. Cette mise au point épistémologique fonde une définition de l'homme comme individu extrême, lieu d'un partage entre la contrainte génétique et un certain degré (le plus important dans le règne animal) de liberté épigénétique. Ainsi résume Prochiantz: «Le cerveau au terme de notre histoire évolutive n'est génétiquement humain que dans son incroyable propension à être modulé dans sa forme même par le processus d'individuation » (p. 144). De fait, la métaphysique et l'éthique sont-elles rejetées hors du 
domaine de la biologie. À leur endroit, l'apport de la biologie est seulement critique : l'irréductible individualité de l'être humain mine en ses fondements l'idée d'une nature et l'annexion de la morale et du droit à cette idée. L'homme se distingue par son « anature" (p. 129), son « anti-nature» (p. 140). L'individuation modifie le statut de la nature humaine. Cette notion ne désigne que les infinies potentialités des individus au sein de l'espèce, potentialités que l'histoire et la culture contribuent à actualiser. L'espèce n'est donc faite que d'individus. L'originalité du propos de Prochiantz est de faire de l'autonomisation de la biologie face à la physique et aux mathématiques la condition de possibilité de l'émancipation du droit et de la morale à l'égard des concepts de nature et de nature humaine. Ce dialogue se veut donc polémique : il s'insurge contre ceux qui souhaiteraient que la science se mêle de métaphysique, contre les anciens sociobiologistes (le darwinisme social) et les actuelles prétentions normatives des sciences cognitives. Le biologiste, se sentant sommé de se prononcer sur les questions éthiques, s'y refuse et fonde en raison ce refus.

Ainsi s'éclaire la référence à Sade. Le genre et la structure du dialogue empruntés à La Philosophie dans le boudoir se justifient par un désir de pédagogie. Les deux premiers tiers de l'ouvrage mettent en place les concepts fondamentaux nécessaires à la compréhension du mécanisme de la création, du maintien et de la reproduction des formes et permettent de « commenter un texte central, théorique, difficile, sans concessions inutiles » (p. 10) intitulé « Biologistes, encore un effort " en écho au sadien « Français, encore un effort si vous voulez être républicains ». Ce texte relie les positions épistémologiques et philosophiques de l'auteur. Plus fondamentalement, Prochiantz se réclame de Sade dans la mesure où celui-ci « trace, le premier, la relation d'identité absolue entre corps et pensée » (p. 11) et où il dénonce le projet de fonder le droit sur une morale prétendument universelle parce que naturelle : « Toute référence à la nature, en tant qu'elle est donnée pour fondement d'une morale ou d'une éthique, est la source de dérèglements terrifiants. Sade en a fait la démonstration par l'absurde en décrivant ce qui se passerait si l'on obéissait aveuglément aux lois de la nature, justifiant par là même la nécessité de lois sociales qui protègent les faibles, mais qui ne sauraient non plus prétendre puiser leur légitimité dans un ordre naturel, encore moins dans un ordre divin, ni intervenir dans ce qui relève du simple dialogue entre soi et soi » (p. 142).

La morale ne précède pas le droit, mais le prolonge et il y a autant de morales que d'individus. L'autonomisation du juridique par rapport au moral garantit le respect de la vie privée des individus et la survie des minorités. Enfin, pour Sade comme pour Prochiantz, la nature humaine qui n'est ni bonne ni mauvaise en soi est penchant vers l'égoïsme et la cruauté. Aussi est-ce parce que la loi s'oppose à cette pente qu'elle est nécessaire. Le répréhensible ne recouvre pas le naturel, mais le passage du fantasme, marque de la jouissance humaine, dans la réalité, la confusion de l'imaginaire et du réel, l'ouverture du boudoir au salon : « La leçon de Sade est qu'il est impossible d'accomplir ce qui hante notre plaisir sans courir à l'extinction de l'espèce » (p. 154).

Le corps du dialogue constitue une initiation à la génétique du développement et articule les concepts de forme, de mémoire et de temps. Prochiantz expose la relation de la morphogenèse et de la mémoire de la position des bourgeons d'organes, l'hypothèse d'une modification des gènes de développement par l'usage des 
organes, le principe de la spatiotemporalité du génome, la relation de la différence des espèces et de la durée d'expression des gènes de développement, les notions de néoténie et de morphogenèse continue du cerveau. Il critique les représentations machinistes et strictement mathématiques du vivant et du cerveau. Il montre que la structure de la partie du génome impliquée dans le développement peut être dite géométrique et non aigébrique. Ainsi, "en postulant que seul le vivant est modèle pour le vivant et en évacuant de la notion de modèle toute valeur de représentation " (p. 73), Prochiantz rejoint un principe fondateur de l'épistémologie canguilhemienne selon lequel la pensée du vivant doit tenir du vivant l'idée du vivant.

Autour de la notion de forme doit se constituer et s'unifier une théorie spécifique du vivant seule susceptible d'affranchir la biologie de l'autorité de la physique et l'éthique de celle de la science : «La question de la forme, de son caractère héréditaire, de son développement fonde toute théorie biologique en tant qu'une telle théorie se doit de rendre compte du vivant comme système capable de se reproduire et d'évoluer" (p. 104). Précisément, les gènes de développement permettant de comprendre les mécanismes développementaux et ouvrant la voie à une évolution expérimentale rendent possible l'unification de la biologie du développement et de la théorie de l'évolution. L'identification de la fonction organogénétique de la nutrition et la définition de la vie comme processus morphogénétique par Claude Bernard qui libère ainsi la biologie du réductionnisme physicaliste et du vitalisme, la loi phylogénétique d'Ernst Haeckel, la découverte de la ségrégation des caractères par Mendel constituent les étapes majeures de l'unification de la biologie autour de la forme. De fait, le projet authentiquement matérialiste en biologie ne consiste pas dans la description du vivant à l'échelle moléculaire et sa subséquente mathématisation, mais dans la question de la reproduction de la forme via celle du génome. L'histoire de la biologie apparaît alors « comme le thêâtre d'une lutte dans la théorie" (p. 108) entre un courant réalisant l'autonomie de la biologie autour des notions de forme et de gènes de développement, et un courant " posant la physique en horizon des sciences du vivant et faisant de la mathématisation le critère ultime de la scientificité » (p. 108). À ce deuxième courant, Prochiantz affilie la compréhension du vivant calquée sur le behaviorisme prônée par le manifeste du Cercle de Vienne (1929), la représentation de la logique et de la construction du cerveau à partir de son assimilation à l'ordinateur et, enfin, les sciences cognitives qu'il définit par leur dévotion à l'empirisme logique, par leur scientificité auto-affirmée, par leur interdisciplinarité prétendue et par le rôle de police des sciences qu'elles s'attribuent.

La critique des sciences cognitives articule les notions de représentation et d'individuation. Concernant la représentation, Prochiantz en appelle à un matérialisme véritable qui affirme l'identité totale de la pensée et du corps. La pensée ne saurait être définie comme un travail sur la représentation, sinon on en revient à un dualisme âme-corps et l'on ne saisit pas la pensée propre au vivant. Au contraire, la pensée est représentation et la représentation est et n'est que corps. Dans un premier temps sont reliés représentation et génome. Les implications morphologiques des mutations génétiques effectuées sur la drosophile et l'identification des gènes homéotiques permettent de montrer que les gènes responsables du développement des organes sont disposés de façon ordonnée le long d'un chromosome chez la drosophile et de quatre chromosomes chez les vertébrés. Cet ordre correspond à celui 
des organes le long de l'axe antéro-postérieur du corps de même que le temps d'expression des gènes correspond au temps auquel les organes sont déterminés dans leur forme. Le développement constitue la projection de la structure spatiotemporelle du génome sur la structure spatio-temporelle du corps. Ainsi « le chromosome contient physiquement une représentation du corps» (p. 115), ce que l'auteur appelle un homonculus. Mais, alors que pour les régions du système nerveux de la moelle épinière à la base du cerveau l'espace morphologique semble bien correspondre à l'espace génétique, un tel isomorphisme topologique entre localisation chromosomique et site d'expression semble perdu au niveau du cerveau. Cependant, il existe bien des homonculus dans le cerveau, mais au lieu d'être génétiques ils sont physiologiques. Ces représentations du corps reflètent l'innervation sensorielle ou motrice de chaque région du corps, c'est-à-dire la taille physiologique des organes; elles sont en rapport physique avec le corps moteur et sensoriel qui, lui, en revanche, est représenté par les quatre homonculus génétiques couchés le long des quatre chromosomes. Prochiantz émet l'hypothèse que " les fibres en provenance du corps sensible sécrètent dans le système nerveux central les protéines à homéodomaine, transportant ainsi l'homonculus génétique de la périphérie vers le cerveau, comme pour l'y imprimer » (p. 122). Le biologiste propose donc, dans un deuxième temps, une définition physiologique de la représentation : «C'est donc l'homonculus qui pense et l'homonculus est corps » (p. 122). Chez l'homme, la forme des homonculus cérébraux, bien qu'enfermée dans le cadre général de l'espèce, ne cesse d'être déformée au rythme de l'histoire individuelle, du fait des afférences sensori-motrices et de l'activité propre des homonculus cérébraux euxmêmes. Ainsi l'adaptation chez l'homme est sélection de gènes permettant d'échapper partiellement à la contrainte génétique. Par conséquent, la représentation permet de penser l'individuation extrême qui définit l'homme et la position matérialiste prend tout son sens. La pensée, forme et déformation, est bien corporelle : le cerveau sécrète la pensée, mais la pensée sécrète elle aussi le cerveau. Ainsi la forme désigne à la fois comme génome la mémoire de l'espèce et comme ensemble des processus physiologiques et épigénétiques de la construction cérébrale la mémoire de l'individu.

Ce parcours initiatique conduisant de l'épistémologie à l'éthique s'adresse paradoxalement à ceux qui, déjà initiés à la biologie mais aussi à la métaphysique, interrogeront à la lumière de leur histoire les concepts proprement philosophiques (par exemple, celui de représentation) que La Biologie dans le boudoir sollicite et élabore de manière originale. 